\title{
Arithmetic properties of polynomial solutions of the Diophantine equation $P(x) x^{n+1}+Q(x)(x+1)^{n+1}=1$
}

\author{
Karl Dilcher ${ }^{1} \cdot$ Maciej Ulas ${ }^{2,3}$
}

Accepted: 25 June 2020 / Published online: 29 April 2021

(c) The Author(s) 2021

\section{Abstract}

For each integer $n \geq 1$ we consider the unique polynomials $P, Q \in \mathbb{Q}[x]$ of smallest degree $n$ that are solutions of the equation $P(x) x^{n+1}+Q(x)(x+1)^{n+1}=1$. We derive numerous properties of these polynomials and their derivatives, including explicit expansions, differential equations, recurrence relations, generating functions, resultants, discriminants, and irreducibility results. We also consider some related polynomials and their properties.

Keywords Recurrence sequence · Polynomial Diophantine equation · Discriminant · Resultant $\cdot$ Generating function

Mathematics Subject Classification Primary 12E10 • 12E05; Secondary 11D04

\section{Introduction}

It is a well-known fact that the Chebyshev polynomials of the first and second kind, $T_{n}(x)$ and $U_{n}(x)$, can be defined as the solutions of the polynomial Pell equation

$$
T_{n}(x)^{2}-\left(x^{2}-1\right) U_{n-1}(x)^{2}=1
$$

in the ring $\mathbb{Z}[x]$; see [1] or [2] for more general polynomial rings.

In this paper we consider the following variant of Eq. (1.1): Since $\mathbb{Q}[x]$ is a Euclidean domain, we know that for given coprime polynomials $f, g \in \mathbb{Q}[x]$ there are polynomials

Research of the first author was supported in part by the Natural Sciences and Engineering Research Council of Canada, Grant \# 145628481.

Maciej Ulas

Maciej.Ulas@im.uj.edu.pl

Karl Dilcher

dilcher@mathstat.dal.ca

1 Department of Mathematics and Statistics, Dalhousie University, Halifax, NS B3H 4R2, Canada

2 Institute of Mathematics of the Polish Academy of Sciences, Świętego Tomasza 30, 31-014 Kraków, Poland

3 Faculty of Mathematics and Computer Science, Institute of Mathematics, Jagiellonian University, Łojasiewicza 6, 30-348 Kraków, Poland 
Table $1 P_{n}(x)$ and $Q_{n}(x)$ for $0 \leq n \leq 4$

\begin{tabular}{lll}
\hline$n$ & $P_{n}(x)$ & $Q_{n}(x)$ \\
\hline 0 & -1 & 1 \\
1 & $2 x+3$ & $-2 x+1$ \\
2 & $-6 x^{2}-15 x-10$ & $6 x^{2}-3 x+1$ \\
3 & $20 x^{3}+70 x^{2}+84 x+35$ & $-20 x^{3}+10 x^{2}-4 x+1$ \\
4 & $-70 x^{4}-315 x^{3}-540 x^{2}-420 x-126$ & $70 x^{4}-35 x^{3}+15 x^{2}-5 x+1$ \\
\hline
\end{tabular}

$P, Q \in \mathbb{Q}[x]$ with $P(x) f(x)+Q(x) g(x)=1$. To make this more specific, we choose $f$ and $g$ to be the simplest pair of coprime polynomials of the same degree, namely $x^{n+1}$ and $(x+1)^{n+1}$, where $n \geq 0$ is an integer. In other words, we consider the equation

$$
P(x) x^{n+1}+Q(x)(x+1)^{n+1}=1 .
$$

If we make the assumption $\operatorname{deg} P \leq n, \operatorname{deg} Q \leq n$, then we have a unique solution $P(x)=$ $P_{n}(x), Q(x)=Q_{n}(x)$ of (1.2). In particular,

$$
P_{n}(x) x^{n+1}+Q_{n}(x)(x+1)^{n+1}=1
$$

for integers $n \geq 0$.

The purpose of this paper is to study properties of the polynomial sequences $P_{n}(x), Q_{n}(x)$. We will see that these polynomials have integer coefficients, are similar to each other, and in spite of some fundamental differences they show some similarities with the Chebyshev polynomials in Eq. (1.1). Moreover, these polynomials appeared in an interesting context of constructing consecutive integers divisible by high powers of their largest prime factors [5, Theorem 4].

The first polynomials $P_{n}(x), Q_{n}(x)$, for $0 \leq n \leq 4$, are shown in Table 1 .

This paper is structured as follows. We begin in Sect. 2 by deriving some basic properties of the polynomials $P_{n}(x)$ and $Q_{n}(x)$, including differential equations, recurrence relations, and generating functions. In Sects. 3 and 4 we consider variants of the original questions concerning the identities (1.2) and (1.3); this will involve the Chebyshev polynomials already mentioned in connection with (1.1). In Sects. 5 and 6 we study resultants and discriminants involving the polynomials $Q_{n}(x)$ and their derivatives. In Sect. 7 we introduce a sequence of polynomials related to the sequence $Q_{n}(x)$ and study their properties. Section 8 is devoted to some irreducibility results, and we conclude this paper with a few further remarks and conjectures in Sect. 9.

\section{Basic properties}

Throughout the rest of this paper, $P_{n}(x)$ and $Q_{n}(x)$ will denote the solutions of the Eq. (1.3).

Proposition 2.1 For any integer $n \geq 0$ we have $\operatorname{deg} P_{n}=\operatorname{deg} Q_{n}$ and

$$
P_{n}(x)=(-1)^{n+1} Q_{n}(-1-x), \quad Q_{n}(x)=(-1)^{n+1} P_{n}(-1-x) .
$$

Proof The definition (1.3) implies that the degrees of $P_{n}$ and $Q_{n}$ must be equal. Now replace $x$ by $-1-x$ in (1.3); then we get

$$
P_{n}(-1-x)(-1)^{n+1}(x+1)^{n+1}+Q_{n}(-1-x)(-1)^{n+1} x^{n+1}=1 .
$$


Now the uniqueness of the solutions of (1.3) implies the two identities in (2.1).

As a consequence of (1.3) and (2.1) we immediately get a few special values.

Corollary 2.2 For any integer $n \geq 0$ we have

$$
P_{n}(-1)=(-1)^{n+1}, \quad P_{n}\left(-\frac{1}{2}\right)=(-1)^{n+1} 2^{n}, \quad Q_{n}\left(-\frac{1}{2}\right)=2^{n}, \quad Q_{n}(0)=1 .
$$

Proof By substituting $x=-1$ in (1.3) we immediately get the first identity, and similarly, $x=0$ gives the fourth identity. Next, if we set $x=-\frac{1}{2}$ in (1.3), we get

$$
(-1)^{n+1} P_{n}\left(-\frac{1}{2}\right)+Q_{n}\left(-\frac{1}{2}\right)=2^{n+1} .
$$

Also, either one of the equations in (2.1), with $x=-\frac{1}{2}$, gives $(-1)^{n+1} P_{n}\left(-\frac{1}{2}\right)=Q_{n}\left(-\frac{1}{2}\right)$. By combining these last two identities we immediately get the third equation in (2.2), and then also the second one.

Another special value will be obtained later in this section. An important consequence of Proposition 2.1 is the fact that it suffices to consider only one of $P_{n}$ and $Q_{n}$. For the rest of this paper we will therefore concentrate on the polynomial sequence $Q_{n}$, and begin by deriving an explicit expression.

Proposition 2.3 For any integer $n \geq 0$ we have $\operatorname{deg} Q_{n}=n$ and

$$
Q_{n}(x)=\sum_{i=0}^{n}(-1)^{i}\left(\begin{array}{c}
n+i \\
i
\end{array}\right) x^{i} .
$$

Before proving this representation, we note that the polynomials $Q_{n}(x)$ have an interesting combinatorial interpretation: the $i$ th coefficient of $Q_{n}(-x)$ counts the numbers of lattice paths from $(0,0)$ to $(n, i)$ using only the steps $(1,0)$ and $(0,1)$; see [12, A046899].

Given the simple form of the representation (2.3), it is not surprising that the polynomials $Q_{n}(-x)$ have been considered before. Using (2.3) as definition, Gould [8] derived some basic properties, including the third identity in (2.2) and two more properties relevant to this paper; those will be mentioned later in this section.

Proof of Proposition 2.3 We differentiate both sides of the identity (1.3), and after some easy manipulation we get

$$
x^{n}\left(x P_{n}^{\prime}(x)+(n+1) P_{n}(x)\right)=-(x+1)^{n}\left((x+1) Q_{n}^{\prime}(x)+(n+1) Q_{n}(x)\right) .
$$

Since $x^{n}$ and $(x+1)^{n}$ are coprime, this last identity implies that $x^{n}$ divides the expression $(x+1) Q_{n}^{\prime}(x)+(n+1) Q_{n}(x)$. Consequently, since deg $Q_{n} \leq n$, we have

$$
(x+1) Q_{n}^{\prime}(x)+(n+1) Q_{n}(x)=c_{n} x^{n}
$$

for some constant $c_{n}$. Now we write

$$
Q_{n}(x)=\sum_{i=0}^{n} q_{i, n} x^{i}
$$

and equate coefficients of $x^{i}$ on both sides of (2.4). First we have by Corollary 2.2 that $q_{0, n}=Q_{n}(0)=1$, and for $i=0,1, \ldots, n-1$ we have

$$
i q_{i, n}+(i+1) q_{i+1, n}+(n+1) q_{i, n}=0,
$$


or equivalently

$$
q_{i+1, n}=-\frac{n+i+1}{i+1} q_{i, n}, \quad i=0,1, \ldots, n-1 .
$$

By iterating this, we immediately get

$$
q_{i, n}=(-1)^{i} \frac{(n+1) \cdots(n+i)}{i !}=(-1)^{i}\left(\begin{array}{c}
n+i \\
i
\end{array}\right) \text { for } i=0,1, \ldots, n .
$$

This with (2.5) completes the proof.

By combining the identities (2.3) and (2.1), we can also obtain an explicit expression for $P_{n}(x)$.

Corollary 2.4 For any integer $n \geq 0$ we have $\operatorname{deg} P_{n}=n$, and

$$
P_{n}(x)=(-1)^{n+1}(2 n+1)\left(\begin{array}{c}
2 n \\
n
\end{array}\right) \sum_{i=0}^{n} \frac{1}{n+i+1}\left(\begin{array}{l}
n \\
i
\end{array}\right) x^{i} .
$$

Proof Combining the first identity in (2.1) with (2.3), we get

$$
\begin{aligned}
(-1)^{n+1} P_{n}(x) & =Q_{n}(-1-x)=\sum_{i=0}^{n}(-1)^{i}\left(\begin{array}{c}
n+i \\
i
\end{array}\right)(-1-x)^{i} \\
& =\sum_{i=0}^{n}\left(\begin{array}{c}
n+i \\
i
\end{array}\right)(x+1)^{i}=\sum_{i=0}^{n}\left(\begin{array}{c}
n+i \\
i
\end{array}\right) \sum_{j=0}^{i}\left(\begin{array}{l}
i \\
j
\end{array}\right) x^{j} \\
& =\sum_{j=0}^{n}\left(\sum_{i=0}^{n}\left(\begin{array}{c}
n+i \\
i
\end{array}\right)\left(\begin{array}{l}
i \\
j
\end{array}\right)\right) x^{j},
\end{aligned}
$$

where we have used the fact that $\left(\begin{array}{l}i \\ j\end{array}\right)=0$ for $j>i$, and then changed the order of summation. Finally we note that the inner sum in the last expansion is known to have the evaluation $\frac{2 n+1}{n+j+1}\left(\begin{array}{c}2 n \\ n\end{array}\right)\left(\begin{array}{l}n \\ j\end{array}\right)$; for instance, after an easy reformulation of the sum, one could use identity (1.48) in [7]. This proves (2.6).

Remark 2.5 The identity (2.6) immediately gives the special values

$$
P_{n}(0)=(-1)^{n+1} \frac{2 n+1}{n+1}\left(\begin{array}{c}
2 n \\
n
\end{array}\right)=(-1)^{n+1}\left(\begin{array}{c}
2 n+1 \\
n+1
\end{array}\right)=(-1)^{n+1} Q_{n}(-1) .
$$

On the other hand, there do not seem to exist explicit formulas for $P_{n}(1)$ and $Q_{n}(1)$. However, the two sequences of their absolute values, namely $(1,5,31,209,1471,10625, \ldots)$ and $(1,1,4,13,46,166, \ldots)$, have some interesting combinatorial interpretations; see the entries A178792 and A026641, respectively, in [12]. Also, it follows from (1.3) that $P_{n}(1)+2^{n+1} Q_{n}(1)=1$.

Remark 2.6 As was observed by one of the referees, to get solutions of the equation $P(x) x^{n+1}+Q(x)(1-x)^{n+1}=1$, one can use the following alternative approach. From the identity

$$
\int_{0}^{1} t^{n}(1-t)^{n} d t=\int_{0}^{x} t^{n}(1-t)^{n} d t+\int_{x}^{1} t^{n}(1-t)^{n} d t
$$


one can observe that that the first term on the right is a polynomial of degree $2 n+1$ divisible by $x^{n+1}$, and the second term is a polynomial divisible by $(1-x)^{n+1}$. Thus, one can use this observation to obtain explicit forms of the polynomials $P, Q$ we are looking for. Moreover, the integral on the left is just $1 /(2 n+1)\left(\begin{array}{c}2 n \\ n\end{array}\right)$, and after multiplication by $(2 n+1)\left(\begin{array}{c}2 n \\ n\end{array}\right)$ the corresponding polynomials are in $\mathbb{Z}[x]$.

Remark 2.7 The polynomial $Q_{n}(x)$ can also be written in terms of a hypergeometric function. Indeed, since $(1+x)^{-n-1}$ is the infinite series of which $Q_{n}(x)$ is the $n$th partial sum, we have

$$
\begin{aligned}
Q_{n}(x) & =\frac{1}{(1+x)^{n+1}}-\sum_{i=n+1}^{\infty}\left(\begin{array}{c}
n+i \\
i
\end{array}\right)(-x)^{i} \\
& =\frac{1}{(1+x)^{n+1}}-(-x)^{n+1} \frac{(2 n+1) !}{(n+1) ! n !} \sum_{i=n+1}^{\infty} \frac{(2 n+2)_{i}(1)_{i}}{(n+2)_{i}} \cdot \frac{(-x)^{i}}{i !},
\end{aligned}
$$

with the Pochhammer symbol $(a)_{i}=a(a+1) \cdots(a+i-1)$ for $i \geq 1$ and $(a)_{0}=1$. Hence,

$$
Q_{n}(x)=\frac{1}{(1+x)^{n+1}}-(-x)^{n+1}\left(\begin{array}{c}
2 n+1 \\
n+1
\end{array}\right){ }_{2} F_{1}(2 n+2,1 ; n+2 ;-x),
$$

however, this representation will not be used further in this paper.

We now return to the identity (2.4) and note that $c_{n}=(2 n+1) q_{n, n}=(-1)^{n}(2 n+1)\left(\begin{array}{c}2 n \\ n\end{array}\right)$, so that

$$
(x+1) Q_{n}^{\prime}(x)+(n+1) Q_{n}(x)=(-1)^{n}(2 n+1)\left(\begin{array}{c}
2 n \\
n
\end{array}\right) x^{n} .
$$

The following generalization of this identity will be useful later. As usual, $Q_{n}^{(k)}(x)$ will denote the $k$ th derivative of $Q_{n}(x)$.

Proposition 2.8 For integers $k$ and $n$ with $1 \leq k \leq n+1$ we have

$$
(x+1) Q_{n}^{(k)}(x)+(n+k) Q_{n}^{(k-1)}(x)=(-1)^{n} \frac{(2 n+1) !}{n !} \frac{x^{n-k+1}}{(n-k+1) !} .
$$

Proof We proceed by induction on $k$. For $k=1$, the identity (2.9) reduces to (2.8). Now we suppose that (2.9) holds for some $k \geq 1$, and differentiate both sides with respect to $x$. Simplifying the resulting identity, we get

$$
(x+1) Q_{n}^{(k+1)}(x)+(n+k+1) Q_{n}^{(k)}(x)=(-1)^{n} \frac{(2 n+1) !}{n !} \frac{x^{n-k}}{(n-k) !},
$$

which is the same as (2.9) with $k$ replaced by $k+1$. This completes the proof by induction.

As a first application of Proposition 2.8 we set $x=-1$ in (2.10), which gives the following special values.

Corollary 2.9 For integers $0 \leq k \leq n$ we have

$$
Q_{n}^{(k)}(-1)=\frac{(-1)^{k}}{n+k+1} \cdot \frac{(2 n+1) !}{n !(n-k) !} .
$$


We note that for $k=0$ this last identity is consistent with the right-hand side of (2.7). As another application of Proposition 2.8 we obtain a homogeneous differential equation satisfied by the polynomials $Q_{n}(x)$.

Proposition 2.10 For $n \geq 0$ we have

$$
x(x+1) Q_{n}^{\prime \prime}(x)+(2 x-n) Q_{n}^{\prime}(x)-n(n+1) Q_{n}(x)=0 .
$$

Proof Using (2.9) with $k=2$ and multiply both sides by $x$, we obtain

$$
x(x+1) Q_{n}^{\prime \prime}(x)+(n+2) x Q_{n}^{\prime}(x)=(-1)^{n} \frac{(2 n+1) !}{n !} \frac{x^{n}}{(n-1) !} .
$$

Then we use (2.9) again, this time with $k=1$, and multiply both sides by $n$, which gives

$$
\left.n(x+1) Q_{n}^{\prime}(x)+n(n+1) Q_{n}(x)\right)=(-1)^{n} \frac{(2 n+1) !}{n !} \frac{x^{n}}{(n-1) !} .
$$

By subtracting this last identity from (2.13), we immediately get (2.12).

Next we present a second-order linear recurrence relation satisfied by the sequence of polynomials $Q_{n}^{(k)}(x)$.

Proposition 2.11 Let $k \geq 0$ be an integer, and set

$$
\begin{aligned}
u_{k, n}(x)= & n(n+k)(x+1)(2(n+k-1) x+n+2 k-1), \\
v_{k, n}(x)= & -2(n+k-1)(2(n+k)-1) x^{2}(2(n+k) x+3 n+4 k) \\
& -(n+2 k-1)(n+2 k)(2(n+k-1) x-n), \\
w_{k, n}(x)= & 2(n+2 k-1)(2(n+k)-1) x(2(n+k) x+n+2 k) .
\end{aligned}
$$

Then the polynomials $Q_{k+n}^{(k)}(x)$ satisfy the recurrence relation

$$
u_{k, n}(x) Q_{k+n}^{(k)}(x)=v_{k, n}(x) Q_{k+n-1}^{(k)}(x)+w_{k, n}(x) Q_{k+n-2}^{(k)}(x) \quad(n \geq 2),
$$

with initial conditions

$$
Q_{k}^{(k)}(x)=(-1)^{k} \frac{(2 k) !}{k !} \text { and } Q_{k+1}^{(k)}(x)=(-1)^{k} \frac{(2 k+1) !}{(k+1) !}(1-2(k+1) x) .
$$

In the special case $k=0$ the expressions $u_{0, n}, v_{0, n}, w_{0, n}$ have the common factor $(2 x+$ 1) $n(n-1)$, and we obtain the following much simpler recurrence relation.

Corollary 2.12 The polynomials $Q_{n}(x)$ satisfy

$$
n(x+1) Q_{n}(x)=-\left(2(2 n-1) x^{2}+2(2 n-1) x-n\right) Q_{n-1}(x)+2(2 n-1) x Q_{n-2}(x)
$$

for $n \geq 2$, with initial conditions $Q_{0}(x)=1$ and $Q_{1}(x)=-2 x+1$.

Proof of Proposition 2.11 By differentiating the explicit formula (2.3), we obtain

$$
Q_{k+n}^{(k)}(x)=\sum_{i=0}^{n}(-1)^{i+k} \frac{(i+k) !}{i !}\left(\begin{array}{c}
n+i+2 k \\
i+k
\end{array}\right) x^{i},
$$

which immediately gives the initial conditions. A straightforward but tedious computation, using (2.16), then shows that the recurrence relation (2.14) is satisfied. We leave the details to the reader. 
Remark 2.13 While the above proof would be sufficient, a few words about the discovery of the recurrence relations are perhaps in order. The relation (2.15) was first guessed with the Maple package EKHAD written by D. Zeilberger, which can be obtained through the online supplement to the book [13]. In particular, the procedure findrec, applied to the explicit formula (2.3), gives the second degree difference operator

$$
\begin{aligned}
& ((1+n) N+2 x(1+2 n))(N(x+1)-1) \\
& \quad=(1+n)(1+x) N^{2}+\left(2(2 n+1) x^{2}+2(2 n+1) x-n-1\right) N-2(1+2 n) x,
\end{aligned}
$$

where $N$ is the forward unit shift operator, i.e., $N\left(f_{n}\right)=f_{n+1}$. Replacing $N^{i}$ by $Q_{n+i}(x)$ and then shifting $n$ to $n-2$ gives the recurrence relation (2.15). The correctness of Zeilberger's algorithm gives an alternative proof that the recurrence is satisfied for all $n$.

Given this nice result, we suspected that similar relations should hold also for the $k$ th derivative of the polynomials $Q_{n+k}(x)$. We checked that for each fixed small $k$ we have a formula similar to (2.15), and then guessed the general form (2.14) from the particular cases.

At this point it should also be mentioned that Gould [8] derived a first-order inhomogeneous recurrence relation for $Q_{n}(-x)$; in our notation, it can be written as

$$
(x+1) Q_{n+1}(x)=Q_{n}(x)+\left(\begin{array}{c}
2 n+1 \\
n+1
\end{array}\right)(2 x+1)(-x)^{n+1} .
$$

This identity was then used in [8] to obtain the following generating function. For the sake of completeness we give a different proof, based on Corollary 2.12.

Proposition 2.14 The ordinary generating function for the polynomials $Q_{n}(x)$ is given by

$$
\frac{1+4 x t+(1+2 x) \sqrt{1+4 x t}}{2(1+x-t)(1+4 x t)}=\sum_{n=0}^{\infty} Q_{n}(x) t^{n} .
$$

Proof We denote the expression on the right of (2.17) by $\mathcal{Q}(x, t)$, and let $\mathcal{Q}^{\prime}(x, t)$ be the derivative with respect to $t$. Then we easily obtain the identities

$$
\begin{aligned}
\sum_{n=0}^{\infty} n Q_{n}(x) t^{n} & =t \mathcal{Q}^{\prime}(x, t), \\
\sum_{n=0}^{\infty} n Q_{n+1}(x) t^{n} & =\frac{1}{t}\left(t \mathcal{Q}^{\prime}(x, t)-\mathcal{Q}(x, t)+1\right), \\
\sum_{n=0}^{\infty} n Q_{n+2}(x) t^{n} & =\frac{1}{t^{2}}\left(t \mathcal{Q}^{\prime}(x, t)-2 \mathcal{Q}(x, t)+2(1-2 x) t\right) .
\end{aligned}
$$

We now use these identities and apply standard methods to transform the recurrence relation (2.14), with $n$ replaced by $n+2$, into the differential equation

$$
\begin{aligned}
& (t-x-1)(4 t x+1)(4 t x-t-1) \mathcal{Q}^{\prime}(x, t) \\
& \quad+\left(6 x t-2 x^{2}-2 x+1\right)((4 x-1) t-1) \mathcal{Q}(x, t)+x((4 x-1) t-1)=0 .
\end{aligned}
$$

Using standard methods for solving first-degree linear differential equations, we get the general solution

$$
\mathcal{Q}(x, t)=\frac{1}{2(1+x-t)}+\frac{c_{1}}{(1+x-t) \sqrt{1+4 x t}} .
$$


The initial condition $\mathcal{Q}^{\prime}(x, 0)=1$ leads to $c_{1}=\frac{1}{2}(2 x+1)$, and we get the desired solution

$$
\mathcal{Q}(x, t)=\frac{1}{2(1+x-t)}\left(\frac{\sqrt{1+4 x t}+2 x+1}{\sqrt{1+4 x t}}\right) .
$$

Finally, we obtain (2.17) by multiplying numerator and denominator of this last equation by $\sqrt{1+4 x t}$.

\section{A Pell-type polynomial equation}

In this section and the next we will consider, more generally, the equation

$$
P_{n}(x) Y(x)^{n+1}+Q_{n}(x) Z(x)^{n+1}=1
$$

for integers $n \geq 0$ and unknown polynomials $Y, Z \in \mathbb{Q}[x]$. We began this paper from the point of view of fixed polynomials $Y(x)=x$ and $Z(x)=x+1$; with the conditions $\operatorname{deg} P_{n} \leq n, \operatorname{deg} Q_{n} \leq n$ this had led us to the unique polynomial sequences $P_{n}, Q_{n}$ in Sects. 1 and 2 .

We now ask the following "inverse question": given our polynomials $P_{n}, Q_{n}$, what can we say about polynomial solutions $Y, Z$ of (3.1)? Are there solutions other than $Y(x)=x$, $Z(x)=x+1$ ?

The case $n=0$ is of no interest since $P_{0}=-1, Q_{0}=1$ implies that for any $Y \in \mathbb{Q}[x]$ (or $\mathbb{Z}[x]$ ) there is a $Z \in \mathbb{Q}[x]$ (or $\mathbb{Z}[x]$ ) satisfying (3.1). Hence we assume $n \geq 1$.

It turns out that the case $n=1$ is of particular interest, and is very different from the case $n \geq 2$. This section will be devoted to this special case; that is, since $P_{1}(x)=2 x+3$ and $Q_{1}(x)=1-2 x$, we consider the equation

$$
(2 x+3) Y(x)^{2}+(1-2 x) Z(x)^{2}=1,
$$

in unknown polynomials $Y, Z \in \mathbb{Q}[x]$.

Proposition 3.1 The Eq. (3.2) has infinitely many solutions which, for $n \geq 0$, are given by

$$
\left\{\begin{array}{l}
Y(x)=Y_{n}(x)=\frac{1}{2}\left(U_{n+1}\left(x+\frac{1}{2}\right)-U_{n}\left(x+\frac{1}{2}\right)\right), \\
Z(x)=Z_{n}(x)=\frac{1}{2}\left(U_{n+1}\left(x+\frac{1}{2}\right)+U_{n}\left(x+\frac{1}{2}\right)\right),
\end{array}\right.
$$

where $U_{n}(y)$ are the Chebyshev polynomials of the second kind. Furthermore, for $n \geq 1$ we have the recurrence relations

$$
\left\{\begin{array}{l}
Y_{n+1}(x)=(2 x+1) Y_{n}(x)-Y_{n-1}(x), \\
Z_{n+1}(x)=(2 x+1) Z_{n}(x)-Z_{n-1}(x),
\end{array}\right.
$$

with initial conditions $Y_{0}(x)=x, Y_{1}(x)=2 x^{2}+x-\frac{1}{2}, Z_{0}(x)=x+1$, and $Z_{1}(x)=$ $2 x^{2}+3 x+\frac{1}{2}$.

Proof We transform the Eq. (3.2) to a Pell-type equation by multiplying both sides by $2 x+3$. Noting that

$$
(2 x-1)(2 x+3)=4\left(\left(x+\frac{1}{2}\right)^{2}-1\right),
$$

we then get the equation

$$
((2 x+3) Y(x))^{2}-\left(\left(x+\frac{1}{2}\right)^{2}-1\right)(2 Z(x))^{2}=2 x+3 .
$$


We now use the following known results from the theory of Pell equations; see, e.g., [11, p. 354] for the case of Pell equations over $\mathbb{Z}$, and [10] for the case of Pell equations over $\mathbb{Z}[x]$.

Suppose that $(P, Q)=\left(r_{0}, s_{0}\right)$ is a solution of the Pell-type equation

$$
P^{2}-d Q^{2}=N
$$

where $d, N \in \mathbb{Z}[x]$, and $d$ is not a perfect square of a polynomial with rational coefficients. Furthermore, suppose that $(P, Q)=\left(P_{n}, Q_{n}\right), n=1,2,3, \ldots$, are the solutions of the Pell equation

$$
P^{2}-d Q^{2}=1
$$

Then the pairs $\left(r_{n}, s_{n}\right)$, defined by

$$
r_{n}+s_{n} \sqrt{d}=\left(r_{0}+s_{0} \sqrt{d}\right)\left(P_{n}+Q_{n} \sqrt{d}\right),
$$

are also solutions of (3.6). We note that the Eqs. (3.6), (3.7) may or may not have solutions in $\mathbb{Z}[x]$. By expanding the right-hand side of (3.8) and equating rational and irrational terms, we immediately get

$$
r_{n}=r_{0} P_{n}+d s_{0} Q_{n}, \quad s_{n}=s_{0} P_{n}+r_{0} Q_{n} .
$$

Comparing (3.5) with (3.6) and noting that $Y(x)=x$ and $Z(x)=x+1$ is a solution of (3.2), we have

$$
r_{0}=x(2 x+3), \quad s_{0}=2(x+2), \quad d=\left(x+\frac{1}{2}\right)^{2}-1, \quad N=2 x+3 .
$$

Furthermore, comparing (3.5) (having 1 instead of $2 x+3$ on the right) with (1.1) (or just invoking [10, Theorem 2]), we see that

$$
P_{n}=T_{n}\left(x+\frac{1}{2}\right), \quad Q_{n}=U_{n-1}\left(x+\frac{1}{2}\right), \quad n=1,2, \ldots
$$

This holds also for $n=0$ since $T_{0}(y)=1$ and, by convention, $U_{-1}(y)=0$. Finally, with $r_{n}=(2 x+3) Y(x)$ and $s_{n}=2 Z(x)$, the identities (3.9), (3.10), (3.11) yield

$$
\left\{\begin{array}{l}
Y(x)=Y_{n}(x)=x T_{n}\left(x+\frac{1}{2}\right)+\frac{1}{2}(2 x-1)(x+1) U_{n-1}\left(x+\frac{1}{2}\right), \\
Z(x)=Z_{n}(x)=(x+1) T_{n}\left(x+\frac{1}{2}\right)+\frac{1}{2} x(2 x+3) U_{n-1}\left(x+\frac{1}{2}\right) .
\end{array}\right.
$$

The initial conditions following (3.4) can be computed from (3.12), using $T_{0}(y)=U_{0}(y)=1$ and $T_{1}(y)=y$. Furthermore, the well-known recurrence relation for the Chebyshev polynomials, namely $T_{n+1}(x)=2 x T_{n}(x)-T_{n-1}(x)$, leads to

$$
T_{n+1}\left(x+\frac{1}{2}\right)=(2 x+1) T_{n}\left(x+\frac{1}{2}\right)-T_{n-1}\left(x+\frac{1}{2}\right),
$$

with the same relation also for the sequence $U_{n}\left(x+\frac{1}{2}\right)$. This means that the polynomial sequences $Y_{n}(x)$ and $Z_{n}(x)$ in (3.12) satisfy this relation as well.

Finally, using the same argument as in the previous paragraph, we see that the right-hand sides of the expressions in (3.3) satisfy (3.4) as well. Using the fact that $U_{1}(y)=2 y$ and $U_{2}(y)=4 y^{2}-1$, we can easily verify that the terms on the right in (3.3) also satisfy the initial conditions following (3.4). This proves the identities in (3.3), and the proof of the the proposition is complete.

We can now use Proposition 3.1 to show that the polynomials $Y_{n}$ and $Z_{n}$ are "almost in $\mathbb{Z}[x]$ ", in the following sense. 
Table $2 Y_{n}(x)$ and $Z_{n}(x)$ for $0 \leq n \leq 4$

\begin{tabular}{lll}
\hline$n$ & $Y_{n}(x)$ & $Z_{n}(x)$ \\
\hline 0 & $x$ & $x+1$ \\
1 & $2 x^{2}+x-\frac{1}{2}$ & $2 x^{2}+3 x+\frac{1}{2}$ \\
2 & $4 x^{3}+4 x^{2}-x-\frac{1}{2}$ & $4 x^{3}+8 x^{2}+3 x-\frac{1}{2}$ \\
3 & $x\left(8 x^{3}+12 x^{2}-3\right)$ & $(x+1)\left(8 x^{3}+12 x^{2}-1\right)$ \\
4 & $16 x^{5}+32 x^{4}+8 x^{3}-10 x^{2}-2 x+\frac{1}{2}$ & $16 x^{5}+48 x^{4}+40 x^{3}+2 x^{2}+6 x-\frac{1}{2}$ \\
\hline
\end{tabular}

Corollary 3.2 Let $n \geq 0$ be an integer. Then

(a) $Y_{n}(x) \in \mathbb{Z}[x]$ and $Z_{n}(x) \in \mathbb{Z}[x]$ when $n \equiv 0(\bmod 3)$;

(b) $Y_{n}(x)+\frac{1}{2} \in \mathbb{Z}[x]$ and $Z_{n}(x)+\frac{1}{2} \in \mathbb{Z}[x]$ when $n \not \equiv 0(\bmod 3)$.

(c) More specifically, we have

$$
Y_{n}(0)=\left\{\begin{array}{ll}
-\frac{1}{2} & \text { if } n \equiv 1,2 \quad(\bmod 6), \\
\frac{1}{2} & \text { if } n \equiv 4,5 \quad(\bmod 6), \\
0 & \text { if } n \equiv 0 \quad(\bmod 3) ;
\end{array} Z_{n}(0)= \begin{cases}-\frac{1}{2} & \text { if } n \equiv 2,4 \quad(\bmod 6), \\
\frac{1}{2} & \text { if } n \equiv 1,5 \quad(\bmod 6), \\
(-1)^{k} & \text { if } n=3 k .\end{cases}\right.
$$

Proof We first note that $Y_{n}$ and $Z_{n}$ satisfy all three statements for $n=0$ and $n=1$. Next, the coefficient $2 x+1$ in the recurrence relations (3.4) guarantees that by induction on $n$ we have that all coefficients of all $Y_{n}, Z_{n}$, with the possible exception of the constant coefficients, are integers.

Finally, it is clear from (3.4) that the constant coefficients of $Y_{n}$ satisfy the recurrence relation $Y_{n+1}(0)=Y_{n}(0)-Y_{n-1}(0)$, and similarly for the sequence $Z_{n}$. Part (c) then follows from a simple induction, and the remaining statements in (a) and (b) also follow.

We observe in Table 2 that both $Y_{3}(x)$ and $Z_{3}(x)$ are reducible. Further computations show that this seems to be the case for all $Y_{3 n}(x)$ and $Z_{3 n}(x)$, a fact confirmed by the following result.

Proposition 3.3 For all integers $n \geq 1$, the polynomials $Y_{3 n}(x)$ and $Z_{3 n}(x)$ are reducible. Specifically, if we set $y:=x+\frac{1}{2}$ for simplicity, we have

$$
\left\{\begin{array}{l}
Y_{3 n}(x)=\frac{1}{2}\left(U_{n}(y)-U_{n-1}(y)\right)\left(U_{2 n+1}(y)-U_{2 n-1}(y)-1\right) \\
Z_{3 n}(x)=\frac{1}{2}\left(U_{n}(y)+U_{n-1}(y)\right)\left(U_{2 n+1}(y)-U_{2 n-1}(y)+1\right)
\end{array}\right.
$$

Proof In view of (3.3), the first identity in (3.13) is equivalent to

$$
U_{3 n+1}(y)-U_{3 n}(y)=\left(U_{n}(y)-U_{n-1}(y)\right)\left(U_{2 n+1}(y)-U_{2 n-1}(y)-1\right) .
$$

We now use the well-known identity

$$
2 T_{j}(y) U_{k}(y)=U_{j+k}(y)-U_{j-k-2}(y), \quad k \leq j-2
$$


(see, e.g., [15]), and set $j=2 n+1, k=0$ and note that $U_{0}(y)=1$. With this, the right-hand side of (3.14) becomes

$$
\begin{aligned}
& \left(U_{n}(y)-U_{n-1}(y)\right)\left(2 T_{2 n+1}(y)-1\right) \\
& \quad=2 T_{2 n+1}(y) U_{n}(y)-2 T_{2 n+1}(y) U_{n-1}(y)-U_{n}(y)+U_{n-1}(y) \\
& =\left(U_{3 n+1}(y)-U_{n-1}(y)\right)-\left(U_{3 n}(y)-U_{n}(y)\right)-U_{n}(y)+U_{n-1}(y) \\
& =U_{3 n+1}(y)-U_{3 n}(y),
\end{aligned}
$$

where in the second equation above we have used (3.15) two more times. This proves (3.14)

For the second part of (3.13), we replace $y$ by $-y$ in (3.14) and use the symmetry property $U_{n}(-y)=(-1)^{n} U_{n}(y)$. Then we get

$$
\begin{aligned}
& (-1)^{n+1} U_{3 n+1}(y)-(-1)^{n} U_{3 n}(y) \\
& \quad=\left((-1)^{n} U_{n}(y)-(-1)^{n-1} U_{n-1}(y)\right)\left(-U_{2 n+1}(y)+U_{2 n-1}(y)-1\right) .
\end{aligned}
$$

Multiplying both sides by $(-1)^{n+1}$, we get

$$
U_{3 n+1}(y)+U_{3 n}(y)=\left(U_{n}(y)+U_{n-1}(y)\right)\left(U_{2 n+1}(y)-U_{2 n-1}(y)+1\right),
$$

which is equivalent to the desired identity.

By iterating the factorizations on the right of (3.13), we obtain the following consequence.

Corollary 3.4 Let $v(k, m):=\frac{1}{2}\left((2 m+1) 3^{k}-1\right)$. Then for all integers $k \geq 0$ and $m \geq 1$, the polynomials $Y_{3 v(k, m)}(x)$ and $Z_{3 v(k, m)}(x)$ have at least $k+2$ nonconstant factors.

Proof By (3.13) it suffices to show that $U_{n}(y) \pm U_{n-1}(y)$ have at least $k+1$ nonconstant factors when $n=v(k, m)$. We fix an $m \geq 1$ and prove this statement by induction on $k$. When $k=0$, then $v(0, m)=m$, and the statement is trivially true. Suppose now that $U_{v(k, m)}(y) \pm U_{v(k, m)-1}(y)$ have at least $k+1$ nonconstant factors. Since $v(k+1, m)=$ $3 v(k, m)+1$, by (3.14), resp. (3.16), we get at least one more irreducible factor in each of the two cases, which completes the proof.

\section{The Eq. (3.1) in general}

In this section we return to the question posed at the beginning of Sect. 3. The following result deals with the general case. As before, $P_{n}$ and $Q_{n}$ denote the polynomials studied in Sects. 1 and 2 , and given by the explicit formulas (2.3) and (2.6).

Proposition 4.1 Let $n \geq 1$ be an integer, and consider the Diophantine equation

$$
P_{n}(x) Y(x)^{n+1}+Q_{n}(x) Z(x)^{n+1}=1
$$

in unknown polynomials $Y, Z$.

(a) If $n=1$, then the Eq. (4.1) has infinitely many solutions $Y, Z \in \mathbb{Q}[x]$.

(b) If $n \geq 2$, then the only solutions $Y, Z \in \mathbb{C}[x]$ of (4.1) are $Y(x)=\zeta x, Z(x)=\xi(x+1)$, where $\zeta$ and $\xi$ are arbitrary $(n+1)$ th roots of unity.

Proof We invoke the Mason-Stothers theorem, which says that the equality $A+B=C$, with $A, B, C \in \mathbb{Q}[x]$ satisfying $\operatorname{gcd}(A, B, C)=1$, implies the inequality

$$
\max \{\operatorname{deg} A, \operatorname{deg} B, \operatorname{deg} C\} \leq N_{0}(A B C)-1 .
$$


For a given $f \in \mathbb{Q}[x]$, the number $N_{0}(f)$ is just the number of different roots of the polynomial $f$; see [17].

We put $A=P_{n} Y^{n+1}, B=Q_{n} Z^{n+1}, C=1$, and $u=\operatorname{deg} Y=\operatorname{deg} Z$. Thus, from the Mason-Stothers theorem we deduce the inequality

$$
n+(n+1) u \leq 2 n+2 u-1 \Longleftrightarrow(n-1) u \leq n-1 .
$$

This last inequality holds only in the following cases:

(1) $n=1$.

(2) $n \geq 2$ and $\operatorname{deg} Y=\operatorname{deg} Z=0$.

(3) $n \geq 2$ and $\operatorname{deg} Y=\operatorname{deg} z=1$.

The case (1) was covered by Proposition 3.1, and leads to part (a) of this proposition. We therefore continue with $\operatorname{deg} Y=\operatorname{deg} Z=0$ and set $Y=a$ and $Z=b$ with $a, b \in \mathbb{C}$. Then (4.1) becomes

$$
P_{n}(x) a^{n+1}+Q_{n}(x) b^{n+1}=1 \quad(n \geq 2) .
$$

We write the expressions (2.6) and (2.3) more explicitly as

$$
\left\{\begin{array}{l}
P_{n}(x)=(-1)^{n+1}\left(\begin{array}{c}
2 n \\
n
\end{array}\right)\left(x^{n}+\left(n+\frac{1}{2}\right) x^{n-1}+\cdots\right), \\
Q_{n}(x)=(-1)^{n+1}\left(\begin{array}{c}
2 n \\
n
\end{array}\right)\left(-x^{n}+\frac{1}{2} x^{n-1}-\cdots\right) .
\end{array}\right.
$$

Using this and equating coefficients of $x^{n+1}$ and of $x^{n}$ in (4.2) we get

$$
a^{n+1}-b^{n+1}=0 \text { and }(2 n+1) a^{n+1}+b^{n+1}=0,
$$

respectively. This system of equations has the unique solution $a=b=0$, which contradicts (4.2). Hence there are no solutions in case (2).

To deal with case (3), we set $Y(x)=a x+c$ and $Z(x)=b x+d$. Then (4.1) becomes

$$
\begin{aligned}
& P_{n}(x)\left(a^{n+1} x^{n+1}+(n+1) a^{n} c x^{n}+\cdots\right) \\
& \quad+Q_{n}(x)\left(b^{n+1} x^{n+1}+(n+1) b^{n} d x^{n}+\cdots\right)=1,
\end{aligned}
$$

where $n \geq 2$. First we equate coefficients of $x^{2 n+1}$ in (4.4), using (4.3). This gives

$$
a^{n+1}=b^{n+1} \quad(a \neq 0) .
$$

Similarly, equating coefficients of $x^{2 n}$ in (4.4), we get

$$
\left(n+\frac{1}{2}\right) a^{n+1}+(n+1) a^{n} c+\frac{1}{2} b^{n+1}-(n+1) b^{n} d=0 .
$$

We now multiply both sides of this last identity by $b$, use (4.5), and divide everything by $(n+1) a^{n}$. This gives

$$
a(b-d)+b c=0 .
$$

Next we show that $c=0$. To do so, we set $x=0$ in (4.1) and use the value of $P(0)$ given in Remark 2.5, along with the fact that $Q_{n}(0)=1$ (see Corollary 2.2). Then we have

$$
(-1)^{n+1}\left(\begin{array}{c}
2 n+1 \\
n+1
\end{array}\right) c^{n+1}+d^{n+1}=1 \quad(n \geq 2) .
$$

First, with $n=2$ and $n=5$, this gives

$$
-\left(\begin{array}{l}
5 \\
3
\end{array}\right) c^{3}+d^{3}=1 \text { and }\left(\begin{array}{c}
11 \\
6
\end{array}\right) c^{6}+d^{6}=1 .
$$


Substituting $d^{3}=1+10 c^{3}$ into the second identity above, we obtain

$$
\left(\left(\begin{array}{c}
11 \\
6
\end{array}\right)+100\right) c^{6}+20 c^{3}=0
$$

which means that either $c=0$, or

$$
\left(\left(\begin{array}{c}
11 \\
6
\end{array}\right)+100\right) c^{3}=-20 .
$$

Second, with $n=3$ and $n=7$ in (4.7), we find in the same way that

$$
\left(\left(\begin{array}{c}
15 \\
8
\end{array}\right)+\left(\begin{array}{l}
7 \\
4
\end{array}\right)^{2}\right) c^{8}-2\left(\begin{array}{l}
7 \\
4
\end{array}\right) c^{4}=0,
$$

which again means that either $c=0$, or $r c^{4}=s$ for certain integers $r, s>0$. But the arguments of the four possible solutions in $c \in \mathbb{C}$ are different from those in (4.8). Therefore we have $c=0$, and consequently $b=d$ by (4.6). This, with (4.5), finally gives part (b) of the result.

To conclude this section, we consider a question related to the original problem concerning the Eq. (1.2). If instead of the polynomials $x$ and $x+1$ we consider an arbitrary fixed pair of coprime polynomials $Y, Z \in \mathbb{Z}[x]$, what can we say about possible solutions of the equation

$$
p_{n}(x) Y(x)^{n+1}+q_{n}(x) Z(x)^{n+1}=1 \quad(n \geq 0),
$$

in unknown polynomials $p_{n}, q_{n} \in \mathbb{Z}[x]$ ? We know that the existence of solutions is not guaranteed since $\mathbb{Z}[x]$ is not a Euclidean domain. However, we have the following result.

Proposition 4.2 Let $Y, Z \in \mathbb{Z}[x]$ be coprime polynomials and suppose that there exist $p_{0}, q_{0} \in \mathbb{Z}[x]$ such that

$$
p_{0}(x) Y(x)+q_{0}(x) Z(x)=1 .
$$

Then for each integer $n \geq 1$ there are polynomials $p_{n}, q_{n} \in \mathbb{Z}[x]$ such that (4.9) holds.

Proof We use the identity $P_{n}(x) x^{n+1}+Q_{n}(x)(x+1)^{n+1}=1$. If we replace $x$ by $-p_{0}(x) Y(x)$, then by (4.10) $1+x$ is replaced by $1-p_{0}(x) Y(x)=q_{0}(x) Z(x)$, and we obtain the desired identity

$$
(-1)^{n+1} P_{n}\left(-p_{0} Y(x)\right) p_{0}^{n+1} Y(x)^{n+1}+Q_{n}\left(-p_{0} Y\right) q_{0}(x)^{n+1} Z(x)^{n+1}=1,
$$

that is,

$$
p_{n}=(-1)^{n+1} P_{n}\left(-p_{0} Y(x)\right) p_{0}^{n+1}, \quad q_{n}=Q_{n}\left(-p_{0} Y\right) q_{0}(x)^{n+1},
$$

and the proof is complete.

Remark 4.3 One can go one step further and look for positive integers $n, m$ and polynomials $Y, Z \in \mathbb{Q}[x]$ satisfying the general equation

$$
P_{n}(x) Y(x)^{m}+Q_{n}(x) Z(x)^{m}=1 .
$$

We were unable to solve this equation. However, we can show that (4.11) has infinitely many solutions. Indeed, if $m \geq 2$ and $n=r m-1$, then $Y(x)=\zeta x^{r}, Z(x)=\xi(x+1)^{r}$ are solutions of (4.11), where $\zeta, \xi$ are arbitrary $m$ th roots of unity. Here we have that $n>m$, 
and this is no coincidence. Indeed, let us assume $m \geq 3$ and differentiate (4.11) with respect to $x$. Then we see that the polynomials $Y, Z$ satisfy the conditions

$$
Z(x)^{m-1}\left|P_{n}^{\prime}(x) Y(x)+m P_{n}(x) Y^{\prime}(x), \quad Y(x)^{m-1}\right| Q_{n}^{\prime}(x) Z(x)+m Q_{n}(x) V^{\prime}(x) .
$$

As a consequence we get the inequalities

$$
(m-1) \operatorname{deg} Z \leq n+\operatorname{deg} Y-1 \text { and }(m-1) \operatorname{deg} Y \leq n+\operatorname{deg} Z-1,
$$

or, equivalently,

$$
\operatorname{deg} Y=\operatorname{deg} Z \leq \frac{(m-1) n}{m(m-2)}
$$

If $n \leq m-2$ then $Y, Z$ are constant polynomials, and so for $n \geq 2$ there are no solutions. If $n=m-1$, then the Eq. (4.11) reduces to (3.1), which was already solved. Thus, $n \geq m$ remains to be considered.

The case $m=2$ leads to polynomial Pell equation $P_{n}(x) Y(x)^{2}+Q_{n}(x) Z(x)^{2}=1$, and for $n \geq 3$ we believe that the only solutions of this equation are of the form $n=2 r-1, Y(x)=$ $\pm x^{r}, Z(x)= \pm(x+1)^{r}$.

\section{The discriminant of $Q_{n}^{(k)}(x)$}

One of the most important invariants of a polynomial is its discriminant. Since we already noted certain similarities between our polynomials $P_{n}(x), Q_{n}(x)$ and the Chebyshev polynomials, we mention the fairly recent papers $[3,6,16,18,19]$ which have dealt with discriminants of Chebyshev-like polynomials.

We begin with recalling the definition of the discriminant. Given a polynomial $f(x)=$ $a_{m} x^{m}+\cdots+a_{1} x+a_{0} \in \mathbb{Q}[x]$ with $a_{m} \neq 0$, the discriminant of $f$ is usually defined by

$$
\operatorname{Disc}(f)=(-1)^{\frac{m(m-1)}{2}} a_{m}^{-1} R\left(f, f^{\prime}\right),
$$

where $f^{\prime}$ is the derivative of $f$ and $R\left(f, f^{\prime}\right)$ is the resultant of $f$ and $f^{\prime}$ which, among other equivalent definitions, can be given by

$$
R\left(f, f^{\prime}\right)=a_{m}^{m-1} \prod_{i=1}^{m} f^{\prime}\left(\theta_{i}\right) .
$$

Here $\theta_{i} \in \mathbb{C}$ is the $i$ th root of $f(x)$. It follows from (5.1) and (5.2) that $\operatorname{Disc}(f)=0$ if and only if $f$ has multiple roots, which is a key property of the discriminant. An alternative definition of the resultant involves a determinant (the Sylvester determinant) whose entries consist only of the coefficients of the two polynomials involved. This implies that $R\left(f, f^{\prime}\right) \in \mathbb{Z}$ if $f \in \mathbb{Z}[x]$.

The following is the main result of this section. As in Sect. 2, we let $Q_{n}^{(k)}(x)$ denote the $k$ th derivative of $Q_{n}(x)$, with $Q_{n}^{(0)}(x)=Q_{n}(x)$.

Theorem 5.1 For all integers $n>k \geq 0$ we have

$$
\operatorname{Disc}\left(Q_{n}^{(k)}(x)\right)=(-1)^{\varepsilon} \frac{n+k+1}{\left(\begin{array}{c}
2 n \\
n+k
\end{array}\right)}\left(\frac{(n+k) !}{(n-k) !}\left(\begin{array}{c}
2 n \\
n
\end{array}\right)(2 n+1)\right)^{n-k-1},
$$


where $\varepsilon:=(n-k)(n-k-1) / 2$. In particular, for $n \geq 1$,

$$
\operatorname{Disc}\left(Q_{n}(x)\right)=(-1)^{\frac{n(n-1)}{2}}(n+1)(2 n+1)^{n-1}\left(\begin{array}{c}
2 n \\
n
\end{array}\right)^{n-2} \text {. }
$$

Proof Let $\psi_{k, i}, 1 \leq i \leq n-k$, be the roots of the polynomial $Q_{n}^{(k)}(x)$. Then we obtain from (2.10),

$$
Q_{n}^{(k+1)}\left(\psi_{k, i}\right)=(-1)^{n} \frac{(2 n+1) !}{(n-k) ! n !} \cdot \frac{\psi_{k, i}^{n-k}}{1+\psi_{k, i}} \quad(1 \leq i \leq n-k) .
$$

With (2.3) we see that the leading coefficient of $Q_{n}^{(k)}(x)$ is

$$
(-1)^{n} \frac{n !}{(n-k) !}\left(\begin{array}{c}
2 n \\
n
\end{array}\right)=(-1)^{n} \frac{(2 n) !}{(n-k) ! n !},
$$

and thus by (5.2) we have

$$
\begin{aligned}
R\left(Q_{n}^{(k)}, Q_{n}^{(k+1)}\right) & =(-1)^{n(n-k-1)}\left(\frac{(2 n) !}{(n-k) ! n !}\right)^{n-k-1} \prod_{i=1}^{n-k} Q_{n}^{(k+1)}\left(\psi_{k, i}\right) \\
& =(-1)^{n}(2 n+1)^{n-k}\left(\frac{(2 n) !}{(n-k) ! n !}\right)^{2(n-k)-1} \prod_{i=1}^{n-k} \frac{\psi_{k, i}^{n-k}}{1+\psi_{k, i}}
\end{aligned}
$$

where we have used (5.5) in the second equation. The leading coefficient (5.6) means that we can write

$$
Q_{n}^{(k)}(x)=(-1)^{n} \frac{(2 n) !}{(n-k) ! n !} \prod_{i=1}^{n-k}\left(x-\psi_{k, i}\right) .
$$

First we set $x=0$ in (5.8) and use the identity $Q_{n}^{(k)}(0)=(-1)^{k}(n+k) ! / n !$, which follows immediately from (2.3). This gives

$$
\prod_{i=1}^{n-k} \psi_{k, i}=\frac{(n-k) !(n+k) !}{(2 n) !}
$$

Next, with $x=-1$ in (5.8) and using the identity (2.11), we get

$$
\prod_{i=1}^{n-k}\left(1+\psi_{k, i}\right)=\frac{2 n+1}{n+k-1} \text {. }
$$

Combining (5.9) and (5.10) with (5.7), we obtain

$$
R\left(Q_{n}^{(k)}, Q_{n}^{(k+1)}\right)=(-1)^{n}(2 n+1)^{n-k} \frac{n+k+1}{2 n+1} \cdot \frac{(n+k) !}{n !}\left(\frac{(n+k) !(2 n) !}{(n-k) ! n !^{2}}\right)^{n-k-1} .
$$

This, with (5.1) and the coefficient (5.6), gives us

$$
\operatorname{Disc}\left(Q_{n}^{(k)}(x)\right)=(-1)^{\varepsilon} \frac{(n+k+1) !(n-k) !}{(2 n+1) !}(2 n+1)^{n-k}\left(\frac{(n+k) !}{(n-k) !}\left(\begin{array}{c}
2 n \\
n
\end{array}\right)\right)^{n-k-1},
$$

with $\varepsilon$ as defined in the statement of the theorem. This last identity immediately implies (5.2), and by setting $k=0$ we get (5.4). 
We briefly turn our attention to the companion polynomial of $Q_{n}(x)$, namely $P_{n}(x)$, and prove the following result.

Corollary 5.2 For all integers $n>k \geq 0$ we have

$$
\operatorname{Disc}\left(P_{n}^{(k)}(x)\right)=\operatorname{Disc}\left(Q_{n}^{(k)}(x)\right) .
$$

Proof For a polynomial $f$ of degree $n$ it was shown in [3, Lemma 4.3] that

$$
\operatorname{Disc}(f(a x+b))=a^{n(n-1)} \operatorname{Disc}(f(x)), \quad \operatorname{Disc}(c f(x))=c^{2(n-1)} \operatorname{Disc}(f(x)),
$$

where $a, b, c$ are constants. We apply these identities to

$$
P_{n}^{(k)}(x)=(-1)^{n+k+1} Q_{n}^{(k)}(-x-1),
$$

which follows from (2.1). Hence we have $a=-1$ and $c= \pm 1$, and since both exponents are even, we immediately get (5.11).

Remark 5.3 Since we have used the Chebyshev polynomials of both kinds earlier in this paper, it is worth mentioning their discriminants:

$$
\operatorname{Disc}\left(T_{n}(x)\right)=2^{(n-1)^{2}} n^{n}, \quad \operatorname{Disc}\left(U_{n}(x)\right)=2^{n^{2}}(n+1)^{n-2} ;
$$

see, e.g., [19].

Knowledge of the discriminant of a polynomial is often important for determining the polynomial's Galois group. In particular, it is known that if the discriminant is the square of a nonzero integer, then the Galois group is a subgroup of the alternating group $A_{n}$; see, e.g., [4, p. 611]. It is therefore of interest to find square discriminants.

Corollary 5.4 Let $n>k \geq 0$ be integers, and set $D_{k, n}:=\operatorname{Disc}\left(Q_{n}^{(k)}\right)$.

(a) If $n \equiv k+2$ or $k+3(\bmod 4)$, then $D_{k, n}$ is not the square of an integer.

(b) If $n \equiv k+1(\bmod 4)$, then for a given $k, D_{k, n}$ is a square for at most finitely many $n$.

(c) If $n \equiv k(\bmod 4)$, then for each $k$ there are infinitely many $n$ such that $D_{k, n}$ is a square.

(d) In particular, $D_{0, n}$ is a square if and only if $n=1$ or $n=n_{j}$, where

$$
n_{j}:=\frac{1}{8}\left((3+2 \sqrt{2})^{2 j+1}+(3-2 \sqrt{2})^{2 j+1}-6\right), \quad j=1,2,3, \ldots
$$

Proof (a) If $n-k \equiv 2$ or $3(\bmod 4)$, then clearly $\varepsilon=(n-k)(n-k-1) / 2$ is odd, and thus $D_{k, n}<0$, which cannot be a square.

(b) If $n-k \equiv 1(\bmod 4)$, then with (5.3) we see that $D_{k, n}$ is a square if and only if

$$
\frac{1}{n+k+1}\left(\begin{array}{c}
2 n \\
n+k
\end{array}\right)=\frac{(2 n)(2 n-1) \cdots(n+k+2)}{(n-k) !}
$$

is a square. However, the prime number theorem implies that for a fixed $k$ and for $n$ sufficiently large, there is always a prime among the members of the sequence $n+k+2, n+k+3, \ldots, 2 n-$ 1 ; this means that (5.13) cannot be a square for these $k$ and $n$, which proves part (b).

(c) If $n-k \equiv 0(\bmod 4)$, then $n-k-2$ is even, and according to (5.3) we consider

$$
\frac{n+k+1}{\left(\begin{array}{c}
2 n \\
n+k
\end{array}\right)} \frac{(n+k) !}{(n-k) !}\left(\begin{array}{c}
2 n \\
n
\end{array}\right)(2 n+1)=(n+k+1)(2 n+1)\left(\frac{(n+k) !}{n !}\right)^{2} \text {, }
$$


where the equality is easily seen by writing the binomial coefficients on the left in terms of factorials. This now implies that $D_{k, n}$ is a square if and only if $(n+k+1)(2 n+1)$ is $\mathrm{s}$ square. If we set $n=4 m+k$, where $m$ is a positive integer, we can write

$$
(4 m+2 k+1)(8 m+2 k+1)=Y^{2}
$$

for some integer $Y \geq 1$. We can write this in the equivalent form

$$
X^{2}-8 Y^{2}=(2 k+1)^{2}
$$

where $X=16 m+6 k+3$. The Eq. (5.14) is a Pell-type equation, and using standard methods for solving such equations (see, e.g., [11, Sect. 7.8]), we find that $X=X_{j}$, where $X_{1}=3(2 k+1), X_{2}=17(2 k+1)$, and $X_{j}=6 X_{j-1}-X_{j-2}$ for $j \geq 3$. From the theory of linear recurrence relations we get the Binet-type formula

$$
X_{j}=\frac{2 k+1}{2}\left((3+2 \sqrt{2})^{j}+(3-2 \sqrt{2})^{j}\right) .
$$

We require $X_{j} \equiv 6 k+3(\bmod 16)$, and an easy calculation shows that this holds if and only if $j$ is odd. The corresponding $n$ that makes $D_{k, n}$ square is then

$$
n=n_{j}=\frac{1}{2}\left(X_{2 j+1}-2 k-3\right)
$$

which proves part (c).

(d) For $k=0$, together with (5.15), this last identity gives (5.12). To complete the proof, we first note that in the case $k=0$ the theory of Pell equations tells us that all solutions of (5.14) are given by (5.15). Returning to part (b) in the case $k=0$, we see that by (5.4) we have $D_{0,1}=1$. Finally, considering again (5.13), there is a prime in the sequence $n+2, n+3, \ldots, 2 n-1$; this follows by Bertrand's Postulate (itself a consequence of the Prime Number Theorem) and the fact that in this case $n+1$ and $2 n$ cannot be prime. The proof is now complete.

Remark 5.5 (a) The first few values of $n>1$ making $D_{0, n}$ a square are

$$
n=24,840,28560,970224,32959080,1119638520,38034750624, \ldots
$$

(b) The statement of part (b) of Corollary 5.4 can be made more explicit by using the following result of Nagura [9]: For $x \geq 25$ there is always a prime $p$ with $x<p<\frac{6}{5} x$.

Setting $x=n+k+1$, an easy calculation shows that whenever $n>\frac{3}{2}(k+1)$, at least one of the integers $n+k+2, \ldots, 2 n-1$ is a prime. This implies that $D_{k, n}$ is not a square when $n \equiv k+1(\bmod 4)$ and $n>\frac{3}{2}(k+1)$. The small cases corresponding to $x<25$ can be eliminated by direct computation. In fact, using the above limit, we verified computationally that $D_{k, n}$ is not a square for any $0 \leq k \leq 5000$ and $n \equiv k+1(\bmod 4)$. The constant $3 / 2$ in the above bound could be lowered by using later improvements of Nagura's result, however at the cost of a higher bound than Nagura's $x \geq 25$.

\section{Resultants involving $Q_{n+k}^{(k)}(x)$}

In the previous section we determined the discriminants, that is, resultants of a polynomial and its derivative. We will now see that the resultant of two consecutive polynomials in our sequence $Q_{n}(x)$ has a particularly easy form. 
Theorem 6.1 For any integer $n \geq 1$ we have

$$
R\left(Q_{n}(x), Q_{n-1}(x)\right)=2^{n}\left(\begin{array}{c}
2 n \\
n
\end{array}\right)^{n-2} .
$$

This result is a consequence of the following theorem. For greater ease of notation, we set

$$
\Delta_{k, n}:=R\left(Q_{n+k}^{(k)}(x), Q_{n-1+k}^{(k)}(x)\right) .
$$

Theorem 6.2 Let $k \geq 0$ be a fixed integer. Then we have

$$
\Delta_{k, n}=\frac{2(2 k+n)}{n}\left(\frac{(2 k+2 n) !}{n !(k+n) !}\right)^{n-2}\left(\frac{(2 k+n-1) !}{(k+n-1) !}\right)^{n} Q_{n-1+k}^{(k)}\left(-\frac{n+2 k}{2(n+k)}\right) .
$$

For $k=0$, the identity $(6.2)$ reduces to

$$
\Delta_{0, n}=2\left(\begin{array}{c}
2 n \\
n
\end{array}\right)^{n-2} Q_{n-1}\left(-\frac{1}{2}\right)=2^{n}\left(\begin{array}{c}
2 n \\
n
\end{array}\right)^{n-2},
$$

where we have used Corollary 2.2 for the second equation. We have thus shown that Theorem 6.1 follows from Theorem 6.2.

Before proving Theorem 6.2, we summarize some useful properties of the resultant. See [3, Sect. 4] for these and more, including references. Suppose we have the following two polynomials:

$$
\left\{\begin{array}{l}
f(x)=a_{0} x^{\mu}+\cdots+a_{\mu-1} x+a_{\mu}=a_{0}\left(x-\alpha_{1}\right) \cdots\left(x-\alpha_{\mu}\right), \\
g(x)=b_{0} x^{m}+\cdots+a_{m-1} x+a_{m}=b_{0}\left(x-\beta_{1}\right) \cdots\left(x-\beta_{m}\right) .
\end{array}\right.
$$

Assuming the variable $x$, and that the resultant is taken with respect to $x$, the following properties hold:

$$
\begin{aligned}
R(f, g) & =a_{0}^{m} \prod_{i=1}^{\mu} g\left(\alpha_{i}\right), \\
R(f, g) & =(-1)^{\mu m} R(g, f), \\
R(f, p q) & =R(f, p) \cdot R(f, q),
\end{aligned}
$$

where $p$ and $q$ are arbitrary polynomials in $x$. Next, if $a$ is a constant and $g$ is a polynomial then, unless $a=g=0$, we have

$$
R(a, g)=R(g, a)=a^{\operatorname{deg} g} .
$$

Finally, we require the following lemma, which can be found as Lemma 4.1 in [3] or, with a different normalization, in [14, p. 58].

Lemma 6.3 Let $f, g$ be as in (6.3). If we can write

$$
f(x)=q(x) g(x)+r(x)
$$

with polynomials $q, r$ and $v:=\operatorname{deg} r$, then

$$
R(g, f)=b_{0}^{\mu-v} R(g, r) .
$$


Proof of Theorem 6.2 We fix $k \geq 0$, and to simplify notation we set

$$
\bar{Q}_{n}(x):=Q_{n+k}^{(k)}(x) \text { and } \Delta_{n}:=\Delta_{k, n} .
$$

Our first goal is to find a recurrence relation between $\Delta_{n}$ and $\Delta_{n-1}$, using the recurrence relation (2.14). For greater ease of notation, we set $u_{n}(x):=u_{k, n}(x), v_{n}(x):=v_{k, n}(x)$, and $w_{n}(x):=w_{k, n}(x)$, and suppress the variable $x$ when there is no danger of confusion.

We apply (6.7) and (6.8) with

$$
f(x)=u_{n}(x) \bar{Q}_{n}(x), \quad g(x)=\bar{Q}_{n-1}(x), \quad r(x)=w_{n}(x) \bar{Q}_{n-2}(x),
$$

and note that $\mu=n+2, v=n$. With explicit expansion (2.16) we get

$$
b_{0}^{\mu-v}=\left(\frac{(n-1+k) !}{(n-1) !}\right)^{2}\left(\begin{array}{c}
2(n-1+k) \\
n-1+k
\end{array}\right)^{2}=: r_{n} .
$$

Then identity (6.8) applied to (2.14) gives us

$$
R\left(\bar{Q}_{n-1}, u_{n} \bar{Q}_{n}\right)=r_{n} R\left(\bar{Q}_{n-1}, w_{n} \bar{Q}_{n-2}\right) .
$$

Now with (6.6) we get

$$
\begin{aligned}
R\left(\bar{Q}_{n-1}, u_{n} \bar{Q}_{n}\right) & =R\left(\bar{Q}_{n-1}, u_{n}\right) R\left(\bar{Q}_{n-1}, \bar{Q}_{n}\right)=R\left(\bar{Q}_{n-1}, u_{n}\right) \Delta_{n}, \\
R\left(\bar{Q}_{n-1}, w_{n} \bar{Q}_{n-2}\right) & =R\left(\bar{Q}_{n-1}, w_{n}\right) R\left(\bar{Q}_{n-1}, \bar{Q}_{n-2}\right)=R\left(\bar{Q}_{n-1}, w_{n}\right) \Delta_{n-1},
\end{aligned}
$$

and identities (6.8) and (6.4) yield, along with the identities in Proposition 2.11,

$$
\begin{aligned}
& R\left(\bar{Q}_{n-1}, u_{n}\right)=(-1)^{n-1} R\left(u_{n}, \bar{Q}_{n-1}\right) \\
& \quad=(-1)^{n-1}(n(n+k))^{n-1} R\left(x+1, \bar{Q}_{n-1}\right) R\left(2(n+k-1) x+n+2 k-1, \bar{Q}_{n-1}\right) \\
& \quad=(-1)^{n-1}(n(n+k))^{n-1} \bar{Q}_{n-1}(-1)(2(n+k-1))^{n-1} \bar{Q}_{n-1}\left(\alpha_{n}\right),
\end{aligned}
$$

where

$$
\alpha_{n}:=\frac{1-n-2 k}{2(n+k-1)} .
$$

Using Corollary 2.9 to evaluate $\bar{Q}_{n-1}(-1)$, we get

$$
R\left(\bar{Q}_{n-1}, u_{n}\right)=(-1)^{n+k-1} p_{n} \cdot \bar{Q}_{n-1}\left(\alpha_{n}\right),
$$

where

$$
p_{n}=\frac{(2 n(n+k-1)(n+k))^{n-1}(2 n+2 k-1) !}{(n-1) !(n+2 k)(n+k-1) !} .
$$

Similarly, we get

$$
\begin{aligned}
& R\left(\bar{Q}_{n-1}, w_{n}\right)=(-1)^{n-1} R\left(w_{n}, \bar{Q}_{n-1}\right) \\
& \quad=(-2(2 n+2 k-1)(n+2 k-1))^{n-1} R\left(x, \bar{Q}_{n-1}\right) R\left((2 n+k) x+n+2 k, \bar{Q}_{n-1}\right), \\
& \quad=(2(2 n+2 k-1)(n+2 k-1))^{n-1} \bar{Q}_{n-1}(0)(2(n+k))^{n-1} \bar{Q}_{n-1}\left(\beta_{n}\right),
\end{aligned}
$$

where

$$
\beta_{n}:=-\frac{n+2 k}{2(n+k)} .
$$


Using (2.16) to evaluate $\bar{Q}_{n-1}(0)$, we get

$$
R\left(\bar{Q}_{n-1}, w_{n}\right)=(-1)^{n+k-1} q_{n} \cdot \bar{Q}_{n-1}\left(\beta_{n}\right),
$$

where

$$
q_{n}=(4(n+k)(2 n+2 k-1)(n+2 k-1))^{n-1} \frac{(n+2 k-1) !}{(n+k-1) !} .
$$

Next, multiplying both sides of (6.14) by $\Delta_{n}$ and combining it with (6.11) and (6.10) we obtain

$$
(-1)^{n+k-1} \Delta_{n} p_{n} \bar{Q}_{n-1}\left(\alpha_{n}\right)=r_{n} R\left(\bar{Q}_{n-1}, w_{n} \bar{Q}_{n-2}\right) .
$$

Similarly, multiplying both sides of (6.17) by $\Delta_{n-1}$ and using (6.12), we get

$$
(-1)^{n+k-1} \Delta_{n-1} q_{n} \bar{Q}_{n-1}\left(\beta_{n}\right)=R\left(\bar{Q}_{n-1}, w_{n} \bar{Q}_{n-2}\right) .
$$

Finally, combining (6.19) and (6.20), we get the desired recurrence relation

$$
\Delta_{n}=\frac{q_{n} r_{n}}{p_{n}} \cdot \frac{\bar{Q}_{n-1}\left(\beta_{n}\right)}{\bar{Q}_{n-1}\left(\alpha_{n}\right)} \Delta_{n-1} \quad(n \geq 2),
$$

with

$$
\Delta_{1}=R\left(\bar{Q}_{1}, \bar{Q}_{0}\right)=R\left((-1)^{k} \frac{(2 k+1) !}{(k+1) !}(1-2(k+1) x),(-1)^{k} \frac{(2 k) !}{k !}\right),
$$

where we have used (2.16). By (6.7), this last resultant evaluates to

$$
\Delta_{1}=(-1)^{k} \frac{(2 k) !}{k !} \text {. }
$$

This completes the first part of the proof.

For the second part of the proof we iterate (6.21) and combine it with (6.22), obtaining

$$
\Delta_{n}=(-1)^{k} \frac{(2 k) !}{k !} \cdot\left(\prod_{j=2}^{n} \frac{q_{j} r_{j}}{p_{j}}\right) \cdot\left(\prod_{j=2}^{n} \frac{\bar{Q}_{j-1}\left(\beta_{j}\right)}{\bar{Q}_{j-1}\left(\alpha_{j}\right)}\right) .
$$

We first deal with the second product in (6.23). Comparing (6.13) with (6.16), we see that $\alpha_{n}=\beta_{n-1}$ for all $n \geq 2$. Hence

$$
\prod_{j=2}^{n} \frac{\bar{Q}_{j-1}\left(\beta_{j}\right)}{\bar{Q}_{j-1}\left(\alpha_{j}\right)}=\prod_{j=2}^{n} \frac{\bar{Q}_{j-1}\left(\beta_{j}\right)}{\bar{Q}_{j-1}\left(\beta_{j-1}\right)}=\frac{\bar{Q}_{n-1}\left(\beta_{n}\right)}{\bar{Q}_{1}\left(\beta_{1}\right)} \cdot \prod_{j=2}^{n-1} \frac{\bar{Q}_{j-1}\left(\beta_{j}\right)}{\bar{Q}_{j}\left(\beta_{j}\right)} .
$$

Since $\beta_{1}=-(2 k+1) / 2(k+1)$, we easily find with $(2.16)$ that

$$
\bar{Q}_{1}\left(\beta_{1}\right)=(-1)^{k} \frac{(2 k+2) !}{(k+1) !} .
$$

Next, the terms in Proposition 2.11 can be shown to evaluate as

$$
u_{n}\left(\beta_{n}\right)=\frac{n^{2} k}{2(n+k)}, \quad v_{n}\left(\beta_{n}\right)=\frac{n k(n+2 k)}{n+k}, \quad w_{n}\left(\beta_{n}\right)=0,
$$

and so the recurrence relation (2.14) gives

$$
\frac{\bar{Q}_{n-1}\left(\beta_{n}\right)}{\bar{Q}_{n-1}\left(\beta_{n-1}\right)}=\frac{n}{2(n+2 k)} \text {. }
$$


This, with (6.24) and (6.25), gives

$$
\prod_{j=2}^{n} \frac{\bar{Q}_{j-1}\left(\beta_{j}\right)}{\bar{Q}_{j-1}\left(\alpha_{j}\right)}=(-1)^{k} \frac{(k+1) !}{(2 k+2) !} \bar{Q}_{n-1}\left(\beta_{n}\right) \prod_{j=2}^{n-1} \frac{j}{2(j+2 k)},
$$

and, with the straightforward evaluation

$$
\prod_{j=2}^{n-1} \frac{j}{2(j+2 k)}=\frac{(n-1) !(2 k+1) !}{2^{n-2}(2 k+n-1) !}
$$

we get

$$
\prod_{j=2}^{n} \frac{\bar{Q}_{j-1}\left(\beta_{j}\right)}{\bar{Q}_{j-1}\left(\alpha_{j}\right)}=(-1)^{k} \frac{(n-1) ! k !}{2^{n-1}(2 k+n-1) !} \bar{Q}_{n-1}\left(\beta_{n}\right) .
$$

Next we deal with the first product in (6.23). We denote it by $\Pi_{n}$, and by combining (6.9), (6.15) and (6.18) we get

$$
\Pi_{n}=\prod_{j=2}^{n} 2^{j-1} \frac{(2 j+2 k-2) !(j+2 k) !}{j !(j+k-1) !^{2}} \cdot \frac{(2 j+2 k-1)^{j-2}(j+2 k-1)^{j-1}}{j^{j-2}(j+k-1)^{j-1}} .
$$

To evaluate this product, we first consider

$$
\prod_{j=2}^{n} j^{j-2}=2^{0} \cdot 3^{1} \cdot 4^{2} \cdots n^{n-2}=\frac{n !}{2 !} \cdot \frac{n !}{3 !} \cdot \frac{n !}{4 !} \cdots \frac{n !}{(n-1) !}
$$

so that

$$
\prod_{j=2}^{n} j^{j-2}=(n !)^{n-2} \prod_{j=2}^{n} \frac{1}{(j-1) !}
$$

Similarly we obtain

$$
\begin{aligned}
& \prod_{j=2}^{n}(j+k-1)^{j-1}=(k+n-1) !^{n-1} \prod_{j=2}^{n} \frac{1}{(j+k-2) !}, \\
& \prod_{j=2}^{n}(j+2 k-1)^{j-1}=(2 k+n-1) !^{n-1} \prod_{j=2}^{n} \frac{1}{(j+2 k-2) !},
\end{aligned}
$$

and with some more effort,

$$
\prod_{j=2}^{n}(2 j+2 k-1)^{j-2}=\left(\frac{(2 k+2 n) !}{(k+n) !}\right)^{n-1} \prod_{j=2}^{n} \frac{(j+k-1) !}{2^{j-1}(2 j+2 k-1) !} .
$$

Substituting (6.28)-(6.31) into (6.27) and simplifying, we get

$$
\Pi_{n}=n !\left(\frac{(2 k+2 n) !(2 k+n-1) !}{n !(k+n) !(k+n-1) !}\right)^{n-1} \prod_{j=2}^{n} \frac{(j+2 k)(j+2 k-1)}{j(2 j+2 k-1)(j+k-1)} .
$$


It is clear that the product on the right of (6.32) can be written as a product and quotient of factorials, and after working out the details, we get

$$
\Pi_{n}=2^{n}\left(\begin{array}{c}
2 k+n \\
n
\end{array}\right)\left(\frac{(2 k+2 n) !}{n !(k+n) !}\right)^{n-2}\left(\frac{(2 k+n-1) !}{(k+n-1) !}\right)^{n} .
$$

Finally, substituting (6.33) and (6.26) into (6.23) and simplifying, we obtain (6.4). The proof is now complete.

As an immediate consequence of Theorem 6.2 we obtain the following result.

Corollary 6.4 For any integers $k \geq 0$ and $n \geq 1$ we have $(-1)^{k} \Delta_{k, n}>0$.

Proof From the explicit expansion (2.16) we see that $(-1)^{k} Q_{k+n-1}^{(k)}(x)>0$ whenever $x<0$. This, together with (6.2), implies the statement.

Our next result, which is rather surprising, but is easy to prove, is similar in nature to Theorem 6.1.

Theorem 6.5 For any $n \geq 0$ we have

$$
R\left(P_{n}(x), Q_{n}(x)\right)=\left(\begin{array}{c}
2 n \\
n
\end{array}\right)^{n+1} .
$$

Proof We rewrite the defining equation (1.3) as

$$
P_{n}(x) x^{n+1}=-(x+1)^{n+1} Q_{n}(x)+1,
$$

and apply Lemma 6.3 with $f(x)=P_{n}(x) x^{n+1}, g(x)=Q_{n}(x)$, and $r(x)=1$. Since $b_{0}=(-1)^{n}\left(\begin{array}{c}2 n \\ n\end{array}\right), \mu=2 n+1$ and $v=0$, we get with (6.8),

$$
R\left(Q_{n}, P_{n} x^{n+1}\right)=(-1)^{n}\left(\begin{array}{c}
2 n \\
n
\end{array}\right)^{n+1} R\left(Q_{n}, 1\right) .
$$

Now, by (6.7) we have $R\left(Q_{n}, 1\right)=1$, and with (6.6), (6.5) and (6.4) we get

$$
\begin{aligned}
R\left(Q_{n}, P_{n} x^{n+1}\right) & =R\left(Q_{n}, P_{n}\right) \cdot R\left(Q_{n}, x^{n+1}\right) \\
& =R\left(Q_{n}, P_{n}\right) \cdot R\left(x^{n+1}, Q_{n}\right)=R\left(Q_{n}, P_{n}\right) \cdot 1 .
\end{aligned}
$$

This, together with (6.35), gives

$$
R\left(Q_{n}, P_{n}\right)=(-1)^{n}\left(\begin{array}{c}
2 n \\
n
\end{array}\right)^{n+1},
$$

and (6.5) finally yields the desired identity (6.34).

\section{The polynomial $Q_{n}(-x)^{2}-Q_{n-1}(-x) Q_{n+1}(-x)$}

Returning to the occasional comparisons we made between the polynomials $P_{n}(x), Q_{n}(x)$ and the Chebyshev polynomials of both kinds, we recall the well-known $2 \times 2$ Hankel determinant expressions

$$
T_{n}(x)^{2}-T_{n-1}(x) T_{n+1}(x)=1-x^{2}, \quad U_{n}(x)^{2}-U_{n-1}(x) U_{n+1}(x)=1,
$$


Table 3 Factorization of $Q_{n}(-x)^{2}-Q_{n-1}(-x) Q_{n+1}(-x), n=1, \ldots, 7$

\begin{tabular}{ll}
\hline$n$ & $Q_{n}(-x)^{2}-Q_{n-1}(-x) Q_{n+1}(-x)$ \\
\hline 1 & $x(1-2 x)$ \\
2 & $x^{2}(1-2 x)(2 x+3)$ \\
3 & $5 x^{3}(1-2 x)\left(2 x^{2}+3 x+2\right)$ \\
4 & $7 x^{4}(1-2 x)\left(10 x^{3}+15 x^{2}+12 x+5\right)$ \\
5 & $42 x^{5}(1-2 x)\left(14 x^{4}+21 x^{3}+18 x^{2}+10 x+3\right)$ \\
6 & $66 x^{6}(1-2 x)\left(84 x^{5}+126 x^{4}+112 x^{3}+70 x^{2}+30 x+7\right)$ \\
7 & $429 x^{7}(1-2 x)\left(132 x^{6}+198 x^{5}+180 x^{4}+120 x^{3}+60 x^{2}+21 x+4\right)$ \\
\hline
\end{tabular}

valid for all integers $n \geq 1$; see, e.g., [15, p. 40]. This immediately gives rise to the question of what can be said about analogous expressions for the polynomials $Q_{n}(x)$. For reasons of simplicity we consider $Q_{n}(-x)$ instead; the first few $2 \times 2$ Hankel determinant expressions, factored over $\mathbb{Q}$, are listed in Table 3 .

The above table strongly suggest that $Q_{n}(-x)^{2}-Q_{n-1}(-x) Q_{n+1}(-x)$ is always divisible by $c_{n} x^{n}(1-2 x)$, where $c_{n} \in \mathbb{Z}$ is a constant depending on $n$, and the co-factor has degree $n-1$ and has positive coefficients. Moreover, it also seems that the sequence of coefficients of the co-factor forms a unimodal sequence. In this section we will show that these properties are true in general. We start with the following.

Proposition 7.1 For integers $n \geq 1$ we define

$$
V_{n}(x):=\frac{2(n+1)}{\left(\begin{array}{c}
2 n \\
n
\end{array}\right) x^{n}(1-2 x)}\left(Q_{n}(-x)^{2}-Q_{n-1}(-x) Q_{n+1}(-x)\right) .
$$

Then the sequence of polynomials $V_{n}(x)$ satisfies the following recurrence relation: $V_{1}(x)=$ $2, V_{2}(x)=2 x+3$, and for $n \geq 3$ we have

$$
n(x-1) V_{n}(x)=(2(2 n-3) x(x-1)-n-1) V_{n-1}(x)+2(2 n-1) x V_{n-2}(x) .
$$

Proof The recurrence relation was guessed with the help of EKHAD (see Remark 2.13), which showed that the recurrence holds for all $n \leq 25$. The proof of correctness for all $n$ is a straightforward but tedious induction using the recurrence relation satisfied by the polynomials $Q_{n}(-x)$. We omit the details.

For integers $n \geq 1$ we now set

$$
V_{n}(x)=\sum_{k=0}^{n-1} a_{k, n} x^{k}
$$

Then the sequences of the first few coefficients are readily identified as $a_{0, n}=n+1$, $a_{1, n}=(n-1) n, a_{2, n}=\frac{1}{2}(n-2)(n-1)(n+1), a_{3, n}=\frac{1}{6}(n-3)(n-2)(n+1)(n+2)$, which are consistent with the entries in Table 3 . These are special cases of the following closed expression for the polynomials $V_{n}(x)$. 
Theorem 7.2 For integers $n \geq 1$ we have

$$
V_{n}(x)=\sum_{k=0}^{n-1} \frac{(n-k)(n-k+1)}{n}\left(\begin{array}{c}
n-1+k \\
k
\end{array}\right) x^{k} .
$$

Proof Following along the lines of the expressions for $a_{0, n}, \ldots, a_{3, n}$ above, it is easy to obtain experimentally a few more cases and then conjecture the form of the coefficients on the right-hand side of (7.4). It can now be verified in a tedious but straightforward way that the right-hand side of (7.4) satisfies the recurrence relation (7.2). Since (7.4) also clearly gives $V_{1}(x)=2$ and $V_{2}(x)=2 x+3$, this proves the theorem.

Using Theorem 7.2, we can easily obtain some properties of the polynomials $V_{n}(x)$. The first one is as follows.

Corollary 7.3 All polynomials $V_{n}(x), n \geq 1$, have positive integer coefficients.

Proof Positivity of the coefficients is clear from (7.4). Now, using the notation of (7.3), we rewrite the coefficients as

$$
a_{k, n}=(n-k)(n-k+1) \frac{(n+k-1)(n+k-2) \cdots(n+1)}{(k-1) ! k} .
$$

The fraction on the right of (7.5) is an integer except when $k \mid n$. But in this case we have $k \mid(n-k)$, so that in either case $a_{k, n}$ is an integer.

Remark 7.4 In the above proof of Corollary 7.3 we actually showed a bit more, namely that $a_{k, n} /(n+1-k)$ is an integer for all $n \geq 1$ and $0 \leq k \leq n-1$.

As another easy consequence of Theorem 7.2 and Proposition 7.1 we get some special values of $V_{n}(x)$.

Corollary 7.5 For all integers $n \geq 1$ we have

$$
V_{n}(0)=n+1, \quad V_{n}\left(\frac{1}{2}\right)=2^{n}, \quad V_{n}(1)=\frac{1}{n+2}\left(\begin{array}{c}
2 n+2 \\
n+1
\end{array}\right)=C_{n+1},
$$

where $C_{n}$ is the nth Catalan number.

Proof The first identity follows immediately from (7.4). For the second and third identities we substitute $x=\frac{1}{2}$ and $x=1$ into (7.2), getting the recurrence relations

$$
\begin{aligned}
n V_{n}\left(\frac{1}{2}\right) & =(4 n-1) V_{n-1}\left(\frac{1}{2}\right)-2(2 n-1) V_{n-2}\left(\frac{1}{2}\right), \\
0 & =-(n+1) V_{n-1}(1)+2(2 n-1) V_{n-2}(1),
\end{aligned}
$$

respectively. The two identities are then obtained by easy inductions.

Next we prove the second observation we made following Table 3. Recall that a polynomial $\sum_{i=0}^{n} a_{i} x^{i}$ is called unimodal if and only if the sequence of its coefficients is unimodal, that is, if there is an integer $m$ (called the mode) with $0 \leq m \leq n$, such that

$$
a_{0} \leq a_{1} \leq \cdots \leq a_{m-1} \leq a_{m} \geq a_{m+1} \geq \cdots \geq a_{n} .
$$

We now state and prove the following result. 
Theorem 7.6 For every integer $n \geq 1$, the polynomial $V_{n}(x)$ is unimodal. More precisely, with the notation (7.3) we have

$$
a_{0, n}<a_{1, n}<\cdots<a_{n-3, n}<a_{n-2, n}>a_{n-1, n} .
$$

Proof Recall that $V_{1}(x)=2$ and $V_{2}(x)=3+2 x$. While for $n=1$ there is nothing to prove, the statement is clearly true for $n=2$. In what follows we therefore assume that $n \geq 3$. First, using (7.3) and (7.4), we get

$$
\frac{a_{n-2, n}}{a_{n-1, n}}=\frac{\frac{6}{n}\left(\begin{array}{c}
2 n-3 \\
n-2
\end{array}\right)}{\frac{2}{n}\left(\begin{array}{c}
2 n-2 \\
n-1
\end{array}\right)}=\frac{3}{2}>1,
$$

where the second equation is easy to verify. Next, for each $k$ with $1 \leq k \leq n-2$, we consider

$$
\frac{a_{k, n}}{a_{k-1, n}}=\frac{(n-k)\left(\begin{array}{c}
n-1+k \\
k
\end{array}\right)}{(n-k+2)\left(\begin{array}{c}
n-2+k \\
k-1
\end{array}\right)}=\frac{(n-k)(n+k-1)}{k(n-k+2)} .
$$

It remains to show that this quotient is greater than 1 , which is equivalent to $(n-k)(n+$ $k-1)>k(n-k+2)$. After an easy manipulation we see that this, in turn, is equivalent to $k(n+1)<n^{2}-n$. But this is true for all $1 \leq k \leq n-2$ since the "worst case" $k=n-2$ leads to the inequality $(n-2)(n+1)<n^{2}-n$, which is clearly true. Thus we have $a_{k-1, n}<a_{k, n}$ for all $1 \leq k \leq n-2$ and $n \geq 3$, which completes the proof.

In analogy to Sect. 2, we can also obtain a generating function for the sequence of polynomials $V_{n}(x)$.

Theorem 7.7 Let

$$
\mathcal{V}(x, t)=\sum_{n=1}^{\infty} V_{n}(x) t^{n}
$$

be the ordinary generating function for the sequence $\left(V_{n}(x)\right)_{n}$. Then

$$
\mathcal{V}(x, t)=\frac{-2 t^{2}+2(2-3 x) t-1+2 x+(1-2 x) \sqrt{1-4 x t}}{2(t+x-1)^{2}} .
$$

Proof We use the same approach as in the proof of Proposition 2.14. Indeed, differentiating both sides of (7.3) with respect to $t$, manipulating the resulting series as we did in the proof of Proposition 2.14 and using the recurrence relation (7.2), we get the differential equation

$$
t(t+x-1)(4 t x-1) \mathcal{V}^{\prime}(x, t)+2 t\left(3 x t-x^{2}+x-1\right) \mathcal{V}(x, t)+2 t(3 x t+x-1)=0 .
$$

Using standard method of solving linear differential equations of degree 1 we easily get the general solution

$$
\mathcal{V}(x, t)=\frac{-2 t^{2}+2(2-3 x) t-1+2 x+2 c_{1} \sqrt{1-4 t x}}{2(t+x-1)^{2}} .
$$

The initial condition $\mathcal{V}(x, 0)=0$ leads to $c_{1}=\frac{1}{2}(1-2 x)$, which finally gives the desired solution (7.4).

We note that there are certain similarities between the generating functions (2.17) and (7.7). In fact, they are related through an identity involving partial derivatives. It can be verified through direct computation. 
Table 4 The polynomials $W_{n}(x), n=1, \ldots, 6$

\begin{tabular}{ll}
\hline$n$ & $W_{n}(x)$ \\
\hline 1 & $x-2 x^{2}$ \\
2 & $3 x^{2}-4 x^{3}-4 x^{4}$ \\
3 & $10 x^{3}-5 x^{4}-20 x^{5}-20 x^{6}$ \\
4 & $35 x^{4}+14 x^{5}-63 x^{6}-140 x^{7}-140 x^{8}$ \\
5 & $126 x^{5}+168 x^{6}-84 x^{7}-630 x^{8}-1176 x^{9}-1176 x^{10}$ \\
6 & $462 x^{6}+1056 x^{7}+660 x^{8}-1848 x^{9}-6468 x^{10}-11088 x^{11}-11088 x^{12}$ \\
\hline
\end{tabular}

Lemma 7.8 Let $\mathcal{R}(x, t):=\mathcal{Q}(-x, t)$. Then

$$
\frac{\partial \mathcal{V}}{\partial t}=x^{2} \frac{\partial^{2} \mathcal{R}}{\partial x^{2}}+2 t \frac{\partial^{2} \mathcal{R}}{\partial t \partial x}+t^{2} \frac{\partial^{2} \mathcal{R}}{\partial t^{2}}+2 x \frac{\partial \mathcal{R}}{\partial x}+4 t \frac{\partial \mathcal{R}}{\partial t}+2 \mathcal{R} .
$$

Remark 7.9 The identity (7.8), along with the explicit formula (2.3) for $Q_{n}(x)$, can be used to give an alternative proof of the explicit formula (7.4) for the polynomial $V_{n}(x)$. We leave the details to the interested reader.

To conclude this section, we return to the original expression of the title, which we denote by

$$
W_{n}(x):=Q_{n}(-x)^{2}-Q_{n-1}(-x) Q_{n+1}(-x)=\sum_{i=0}^{2 n} w_{i, n} x^{i} .
$$

The first few of these polynomials are listed in Table 4.

The following properties of the polynomials $W_{n}(x)$ are an immediate consequence of (7.1) and Theorem 7.2.

Corollary 7.10 For all integers $n \geq 1$ and $0 \leq j \leq n-1$ we have $w_{j, n}=0$, and

$$
w_{n, n}=\frac{1}{2}\left(\begin{array}{c}
2 n \\
n
\end{array}\right), \quad w_{2 n-1, n}=w_{2 n, n}=-2 C_{n-1} C_{n},
$$

where $C_{n}=\frac{1}{n+1}\left(\begin{array}{c}2 n \\ n\end{array}\right)$ is the $n$th Catalan number.

To motivate the last result of this section, we consider the entry for $n=4$ in Table 4 and note that

$$
\frac{14}{2}-\frac{63}{4}-\frac{140}{8}-\frac{140}{16}=-35
$$

This is actually no surprise since by (7.1) we have $W_{n}\left(\frac{1}{2}\right)=0$ for all $n \geq 1$ and thus, by (7.9) we have

$$
\sum_{j=1}^{n} w_{n+j, n} 2^{-j}=-w_{n, n}=-\frac{1}{2}\left(\begin{array}{c}
2 n \\
n
\end{array}\right) \quad(n \geq 1) .
$$

This identity is a special case of the following result. 
Proposition 7.11 For integers $n \geq 1$ and $0 \leq i \leq n-1$ we have

$$
\sum_{j=i+1}^{n} w_{n+j, n} 2^{i-j}=-\frac{(n-i)(n-i+1)}{2 n(n+1)}\left(\begin{array}{c}
2 n \\
n
\end{array}\right)\left(\begin{array}{c}
n-1+i \\
i
\end{array}\right) .
$$

Before proving this identity we note that in the two extreme cases we get (7.11) when $i=0$, and the right-most equation in (7.10) when $i=n-1$.

Proof of Proposition 7.11 We first prove the identity

$$
\frac{W_{n}(x)}{x^{n}}=\sum_{i=0}^{n} w_{n+i, n} x^{i}=(2 x-1) \sum_{i=0}^{n-1}\left(\sum_{j=i+1}^{n} \frac{w_{n+j, n}}{2^{j-i}}\right) x^{i},
$$

where the left equation comes from (7.9). To do so, we denote the right-most term of (7.13) by $R_{n}(x)$ and manipulate the double sum as follows:

$$
\begin{aligned}
R_{n}(x) & =\sum_{i=0}^{n-1}\left(\sum_{j=i+1}^{n} \frac{w_{n+j, n}}{2^{j-i-1}}\right) x^{i+1}-\sum_{i=0}^{n-1}\left(\sum_{j=i+1}^{n} \frac{w_{n+j, n}}{2^{j-i}}\right) x^{i} \\
& =\sum_{i=1}^{n}\left(\sum_{j=i}^{n} \frac{w_{n+j, n}}{2^{j-i}}\right) x^{i}-\sum_{i=0}^{n-1}\left(\sum_{j=i+1}^{n} \frac{w_{n+j, n}}{2^{j-i}}\right) x^{i} \\
& =\sum_{i=0}^{n}\left(\sum_{j=i}^{n} \frac{w_{n+j, n}}{2^{j-i}}\right) x^{i}-\sum_{j=0}^{n} \frac{w_{n+j, n}}{2^{j}}-\sum_{i=0}^{n}\left(\sum_{j=i+1}^{n} \frac{w_{n+j, n}}{2^{j-i}}\right) x^{i} \\
& =\sum_{i=0}^{n} w_{n+i, n} x^{i}-\sum_{j=0}^{n} w_{n+j, n}\left(\frac{1}{2}\right)^{j}
\end{aligned}
$$

where we have combined the first and third sum from the second-last line. The second sum in the last line then vanishes since $W_{n}\left(\frac{1}{2}\right)=0$ for all $n \geq 0$, and the proof of (7.13) is complete.

Now we rewrite (7.1), with (7.9), as

$$
\frac{1}{2(n+1)}\left(\begin{array}{c}
2 n \\
n
\end{array}\right) V_{n}(x)=\frac{W_{n}(x)}{x^{n}(1-2 x)}=-\sum_{i=0}^{n-1}\left(\sum_{j=i+1}^{n} \frac{w_{n+j, n}}{2^{j-i}}\right) x^{i} .
$$

Finally, using (7.4) and equating coefficients of $x^{i}, 0 \leq i \leq n-1$, we get (7.12).

\section{Some irreducibility results}

In this brief section we prove some irreducibility results for the main objects of study in this paper, namely the polynomials $Q_{n}(x)$ and their derivatives, and the polynomials $V_{n}(x)$.

Theorem 8.1 Let $n \geq 1$ and $0 \leq k \leq n-1$ be integers. Then the polynomial $Q_{n}^{(k)}(x)$ is irreducible over $\mathbb{Q}$ when $n+k+1$ is a prime, or when $2 n+1$ is a prime. 
Proof To prove the first statement, we show that $Q_{p-k-1}^{(k)}(x)$ is $p$-Eisenstein. To do so, we rewrite the explicit expression (2.16) as

$$
Q_{n}^{(k)}(x)=\frac{(-1)^{k}}{n !} \sum_{i=0}^{n-k}(-1)^{i}(n+k+i) ! \frac{x^{i}}{i !},
$$

so the coefficients of $Q_{p-k-1}^{(k)}(x)$ are

$$
\frac{(n+k+i) !}{n ! i !}=\frac{(p+i-1) !}{(p-k-1) ! i !}, \quad i=0,1, \ldots, p-2 k-1 .
$$

We note that for each positive index $i$ in this range the numerator of the last fraction is divisible by $p$, but not by $p^{2}$, while the factorials $(p-k-1) !$ and $i$ ! in the denominator are not divisible by $p$. It is also clear that the constant coefficient, $(p-1) ! /(p-k-1)$ !, is not divisible by $p$. Hence the polynomial $Q_{p-k-1}^{(k)}(x)$ is $p$-Eisenstein.

For the second statement, we assume that $p:=2 n+1$ is prime, and show that $Q_{n}^{(k)}(-x-1)$ is $p$-Eisenstein, which would imply irreducibility of $Q_{n}^{(k)}(x)$. For this purpose we combine (2.1) with (2.6), and upon taking the $k$ th derivative we get

$$
Q_{n}^{(k)}(-x-1)=(-1)^{(2 n+1)}\left(\begin{array}{c}
2 n \\
n
\end{array}\right) \sum_{i=k}^{n} \frac{1}{n+i+1} \cdot \frac{n !}{(i-k) !(n-1) !} x^{i-k} .
$$

We know that this polynomial has integer coefficients, and we see that for each index $i$ with $k \leq i \leq n-1$ the corresponding coefficient is divisible by the prime $p=2 n+1$, but not by $p^{2}$. For $i=n$, however, we have cancellation, and thus the leading coefficient is not divisible by $p$. Hence $Q_{n}^{(k)}(-x-1)$ is $p$-Eisenstein, and the proof is complete.

Theorem 8.2 The polynomial $V_{n}(x)$ is irreducible over $\mathbb{Q}$ if $2 n+1$ is prime.

Proof We set again $p:=2 n+1$ and show that $V_{n}(x+1)$ is $p$-Eisenstein. To do so, we use (7.4) and a binomial expansion, followed by changing the order of summation:

$$
\begin{aligned}
& V_{n}(x+1) \\
& \quad=\sum_{k=0}^{n-1} \frac{(n-k)(n-k+1)}{n}\left(\begin{array}{c}
n-1+k \\
k
\end{array}\right) \sum_{j=0}^{k}\left(\begin{array}{l}
k \\
j
\end{array}\right) x^{j} \\
& \quad=\sum_{j=0}^{n-1}\left(\sum_{k=j}^{n-1} \frac{(n-k)(n-k+1)}{n}\left(\begin{array}{c}
n-1+k \\
k
\end{array}\right)\left(\begin{array}{l}
k \\
j
\end{array}\right)\right) x^{j} \\
& =\sum_{j=0}^{n-1}\left(\frac{(n-1+j) !}{n ! j !} \sum_{k=0}^{n-1-j}(n-k-j)(n-k-j+1)\left(\begin{array}{c}
n-1+k+j \\
k
\end{array}\right)\right) x^{j},
\end{aligned}
$$

where the last line results from a straightforward manipulation of the binomial coefficients in the previous line. The inner sum in this last line can be evaluated by various means, including the function sum in Maple which, after some manipulations, gives

$$
V_{n}(x+1)=\sum_{j=0}^{n-1} \frac{2}{n+j}\left(\begin{array}{c}
n+j \\
n
\end{array}\right)\left(\begin{array}{c}
2 n+1 \\
n+j+2
\end{array}\right) x^{j} .
$$


As in the proof of the previous theorem we observe that the coefficients of $V_{n}(x+1)$ are integers, and that for $0 \leq j \leq n-2$ they are all divisible by $p=2 n+1$, but not by $p^{2}$, and that the coefficient of $x^{n-1}$ is not divisible by $p$. Hence $V_{n}(x+1)$ is $p$-Eisenstein, as claimed.

\section{Further remarks and conjectures}

In this final section we collect some further remarks and conjectures related to the objects studied in this paper.

1. In Sect. 5 we already mentioned the fact that a polynomial in $\mathbb{Z}[x]$ with a square discriminant has its Galois group contained in the alternating group $A_{n}$. Can anything more be said about the Galois group of $Q_{n}$ ? Computations support the following conjecture, where we set $D_{0, n}=\operatorname{Disc}\left(Q_{n}\right)$, as in Corollary 5.4.

Conjecture 9.1 For integers $n \geq 2$ we have

$$
\operatorname{Gal}\left(Q_{n}\right)= \begin{cases}A_{n} & \text { if } D_{0, n} \text { is a square, } \\ S_{n} & \text { if } D_{0, n} \text { is not a square. }\end{cases}
$$

2. In Sect. 8 we proved some partial irreducibility results. However, computations indicate that much more is true.

Conjecture 9.2 For all integers $n \geq 1$ and $0 \leq k \leq n-1$ the polynomials $Q_{n}^{(k)}(x)$ and $V_{n}(x)$ are irreducible over $\mathbb{Q}$.

3. It is clear that Proposition 4.2 is in fact true in greater generality. More precisely, if $R$ is a commutative ring with $0 \neq 1$ and $p_{0}, q_{0}, Y, Z \in R$ satisfy the identity $p_{0} Y+q_{0} Z=1$, then there are $p_{n}, q_{n} \in R$ such that $p_{n} Y^{n+1}+q_{n} Z^{n+1}=1$. Indeed, the proof of this more general statement is exactly the same as that of Proposition 4.2.

This gives rise to the question of whether such a result is also true in a non-commutative setting. We don't know the answer and formulate the following open

Question 9.3 Let $R$ be a non-commutative ring and suppose that $p_{0}, q_{0}, Y, Z$ satisfy the equation $p_{0} Y+q_{0} Z=1$, where $Y, Z$ are not nilpotent elements. Given a fixed positive integer $n$, do there exist $p_{n}, q_{n} \in R$ such that $p_{n} Y^{n+1}+q_{n} Z^{n+1}=1$ ?

If $Y, Z$ are allowed to be nilpotent elements in $R$, it is easy to show that in general we cannot expect a positive answer. Indeed, let $R=M_{2,2}$ be the ring of $2 \times 2$ matrices with integer coefficients and consider the identity $p_{0} Y+q_{0} Z=I$, where

$$
p_{0}=\left(\begin{array}{ll}
0 & 0 \\
1 & 0
\end{array}\right), Y=\left(\begin{array}{ll}
0 & 1 \\
0 & 0
\end{array}\right), q_{0}=\left(\begin{array}{ll}
0 & 1 \\
0 & 0
\end{array}\right), Z=\left(\begin{array}{ll}
0 & 0 \\
1 & 0
\end{array}\right)
$$

However, we have $Y^{2}=Z^{2}=0$ and thus, for each $n \geq 1$ and any $p, q \in R$ we have $p Y^{n+1}+q Z^{n+1} \neq I$.

Acknowledgements The authors express their gratitude to the anonymous referees for constructive suggestions which improved the quality of the paper. 
Open Access This article is licensed under a Creative Commons Attribution 4.0 International License, which permits use, sharing, adaptation, distribution and reproduction in any medium or format, as long as you give appropriate credit to the original author(s) and the source, provide a link to the Creative Commons licence, and indicate if changes were made. The images or other third party material in this article are included in the article's Creative Commons licence, unless indicated otherwise in a credit line to the material. If material is not included in the article's Creative Commons licence and your intended use is not permitted by statutory regulation or exceeds the permitted use, you will need to obtain permission directly from the copyright holder. To view a copy of this licence, visit http://creativecommons.org/licenses/by/4.0/.

\section{References}

1. M. Davis, H. Putnam, Diophantine sets over polynomial rings. Ill. J. Math. 7, 251-256 (1963)

2. J. Denef, The Diophantine problem for polynomial rings and fields of rational functions. Trans. Am. Math. Soc. 242, 391-399 (1978)

3. K. Dilcher, K.B. Stolarsky, Resultants and discriminants of Chebyshev and related polynomials. Trans. Am. Math. Soc. 357(3), 965-981 (2005)

4. D.S. Dummit, R.M. Foote, Abstract Algebra, 3rd edn. (Wiley, Hoboken, 2004)

5. J.-M. de Koninck, M. Moineau, Consecutive integers divisible by a power of their largest prime factor. J. Integer Seq. 21, Article 18.9.3 (2018)

6. J. Gishe, M.E.H. Ismail, Resultants of Chebyshev polynomials. J. Anal. Appl. 27(4), 499-508 (2008)

7. H.W. Gould, Combinatorial Identities, revised edn. (Gould Publications, Morgantown, 1972)

8. H.W. Gould, A class of binomial sums and a series transform. Util. Math. 45, 71-83 (1994)

9. J. Nagura, On the interval containing at least one prime number. Proc. Jpn. Acad. 28, 177-181 (1952)

10. M.B. Nathanson, Polynomial Pell's equations. Proc. Am. Math. Soc. 56, 89-92 (1976)

11. I. Niven, H.S. Zuckerman, H.L. Montgomery, An Introduction to the Theory of Numbers, 5th edn. (Wiley, Hoboken, 1991)

12. OEIS Foundation Inc. (2011), The On-Line Encyclopedia of Integer Sequences, http://oeis.org. Accessed 11 Jan 2020

13. M. Petkovšek, H. Wilf, D. Zeilberger, A=B, A K Peters/CRC Press, 1996. Homepage for this book, https:// www.math.upenn.edu/ wilf/AeqB.html. Accessed 11 Jan 2020

14. M. Pohst, H. Zassenhaus, Algorithmic Algebraic Number Theory (Cambridge University Press, Cambridge, 1989)

15. T.J. Rivlin, Chebyshev Polynomials. From Approximation Theory to Algebra and Number Theory, 2nd edn. (Wiley, New York, 1990)

16. K.B. Stolarsky, Discriminants and divisibility for Chebyshev-like polynomials, in Number Theory for the Millennium, III (Urbana, 2000), pp. 243-252 (A K Peters, Natick, 2002)

17. W.W. Stothers, Polynomial identities and Hauptmoduln. Q. J. Math. Oxf. Ser. (2) 32, 349-370 (1981)

18. K. Tran, Discriminants of Chebyshev-like polynomials and their generating functions. Proc. Am. Math. Soc. 137(10), 3259-3269 (2009)

19. K. Tran, Discriminants of polynomials related to Chebyshev polynomials: the "Mutt and Jeff" syndrome. J. Math. Anal. Appl. 383(1), 120-129 (2011)

Publisher's Note Springer Nature remains neutral with regard to jurisdictional claims in published maps and institutional affiliations. 